\title{
Measurements of the absolute branching fractions of $D^{0(+)} \rightarrow K \bar{K} \pi \pi$ decays
}

M. Ablikim, ${ }^{1}$ M. N. Achasov, ${ }^{10, c}$ P. Adlarson, ${ }^{64}$ S. Ahmed, ${ }^{15}$ M. Albrecht, ${ }^{4}$ R. Aliberti, ${ }^{28}$ A. Amoroso, ${ }^{63 a, 63 c}$ Q. An,${ }^{60,48}$ Anita, ${ }^{21}$ X. H. Bai,${ }^{54}$ Y. Bai, ${ }^{47}$ O. Bakina, ${ }^{29}$ R. Baldini Ferroli, ${ }^{23 a}$ I. Balossino, ${ }^{24 a}$ Y. Ban, ${ }^{38, k}$ K. Begzsuren, ${ }^{26}$ J. V. Bennett, ${ }^{5}$ N. Berger ${ }^{28}$ M. Bertani, ${ }^{23 \mathrm{a}}$ D. Bettoni, ${ }^{24 \mathrm{a}} \mathrm{F}$. Bianchi, ${ }^{63 \mathrm{a}, 63 \mathrm{c}} \mathrm{J}$. Biernat ${ }^{64} \mathrm{~J}$. Bloms, ${ }^{57}$ A. Bortone ${ }^{63 \mathrm{a}, 63 \mathrm{c}} \mathrm{I}$. Boyko, ${ }^{29}$ R. A. Briere ${ }^{5}$ H. Cai,${ }^{65}$ X. Cai,${ }^{1,48}$ A. Calcaterra, ${ }^{23 a}$ G. F. Cao, ${ }^{1,52}$ N. Cao, ${ }^{1,52}$ S. A. Cetin, ${ }^{51 b}$ J. F. Chang, ${ }^{1,48}$ W. L. Chang, ${ }^{1,52}$ G. Chelkov, ${ }^{29, b}$ D. Y. Chen, ${ }^{6}$ G. Chen, ${ }^{1}$ H. S. Chen, ${ }^{1,52}$ M. L. Chen, ${ }^{1,48}$ S. J. Chen, ${ }^{36}$ X. R. Chen, ${ }^{25}$ Y. B. Chen, ${ }^{1,48}$ Z. J. Chen, ${ }^{20,1}$ W. S. Cheng, ${ }^{63 c}$ G. Cibinetto, ${ }^{24 a}$ F. Cossio, ${ }^{63 c}$ X. F. Cui,${ }^{37}$ H. L. Dai, ${ }^{1,48}$ J. P. Dai, ${ }^{42, g}$ X. C. Dai, ${ }^{1,52}$

A. Dbeyssi, ${ }^{15}$ R. B. de Boer, ${ }^{4}$ D. Dedovich ${ }^{29}$ Z. Y. Deng, ${ }^{1}$ A. Denig, ${ }^{28}$ I. Denysenko, ${ }^{29}$ M. Destefanis, ${ }^{63 a, 63 c}$

F. De Mori, ${ }^{63 a, 63 c}$ Y. Ding, ${ }^{34}$ C. Dong, ${ }^{37}$ J. Dong, ${ }^{1,48}$ L. Y. Dong, ${ }^{1,52}$ M. Y. Dong, ${ }^{1,48,52}$ S. X. Du, ${ }^{68}$ J. Fang, ${ }^{1,48}$ S. S. Fang, ${ }^{1,52}$ Y. Fang, ${ }_{1}^{1}$ R. Farinelli, ${ }^{24 a}$ L. Fava, ${ }^{63 b, 63 c}$ F. Feldbauer, ${ }^{4}$ G. Felici, ${ }^{23 a}$ C. Q. Feng, ${ }^{60,48}$ M. Fritsch, ${ }^{4}$ C. D. Fu, ${ }^{1}$ Y. Fu, ${ }^{1}$ X. L. Gao, ${ }^{60,48}$ Y. Gao ${ }^{61}$ Y. Gao ${ }^{38, k}$ Y. G. Gao, ${ }^{6}$ I. Garzia, ${ }^{24 a, 24 b}$ E. M. Gersabeck ${ }^{55}$ A. Gilman,${ }^{56}$ K. Goetzen, ${ }^{11}$ L. Gong, ${ }^{37}$ W. X. Gong, ${ }^{1,48}$ W. Gradl, ${ }^{28}$ M. Greco, ${ }^{63 a, 63 c}$ L. M. Gu, ${ }^{36}$ M. H. Gu, ${ }^{1,48}$ S. Gu, ${ }^{2}$ Y. T. Gu, ${ }^{13}$ C. Y. Guan, ${ }^{1,52}$ A. Q. Guo, ${ }^{22}$ L. B. Guo ${ }^{35}$ R. P. Guo ${ }^{40}$ Y. P. Guo, ${ }^{9, h}$ Y. P. Guo, ${ }^{28}$ A. Guskov, ${ }^{29}$ S. Han,${ }^{65}$ T. T. Han, ${ }^{41}$ T. Z. Han, ${ }^{9, h}$ X. Q. Hao, ${ }^{16}$

F. A. Harris, ${ }^{53}$ K. L. He ${ }^{1,52}$ F. H. Heinsius, ${ }^{4}$ C. H. Heinz ${ }^{28}$ T. Held, ${ }^{4}$ Y. K. Heng, ${ }^{1,48,52}$ M. Himmelreich, ${ }^{11, f}$ T. Holtmann, ${ }^{4}$ Y. R. Hou ${ }^{52}$ Z. L. Hou, ${ }^{1}$ H. M. Hu, ${ }^{1,52}$ J. F. Hu,${ }^{42,9}$ T. Hu, ${ }^{1,48,52}$ Y. Hu, ${ }^{1}$ G. S. Huang, ${ }^{60,48}$ L. Q. Huang, ${ }^{61}$ X. T. Huang ${ }^{41}$ Y. P. Huang, ${ }^{1}$ Z. Huang, ${ }^{38, k}$ N. Huesken ${ }^{57}$ T. Hussain, ${ }^{62}$ W. Ikegami Andersson, ${ }^{64}$ W. Imoehl, ${ }^{22}$ M. Irshad, ${ }^{60,48}$ S. Jaeger, ${ }^{4}$ S. Janchiv, ${ }^{26, j}$ Q. Ji, ${ }^{1}$ Q. P. Ji, ${ }^{16}$ X. B. Ji, ${ }^{1,52}$ X. L. Ji, ${ }^{1,48}$ H. B. Jiang, ${ }^{41}$ X. S. Jiang, ${ }^{1,48,52}$ X. Y. Jiang, ${ }^{37}$ J. B. Jiao, ${ }^{41}$ Z. Jiao ${ }^{18}$ S. Jin, ${ }^{36}$ Y. Jin,${ }^{54}$ T. Johansson, ${ }^{64}$ N. Kalantar-Nayestanaki, ${ }^{31}$ X. S. Kang,${ }^{34}$ R. Kappert, ${ }^{31}$ M. Kavatsyuk ${ }^{31}$ B. C. Ke, ${ }^{43,1}$ I. K. Keshk, ${ }^{4}$ A. Khoukaz, ${ }^{57}$ P. Kiese,${ }^{28}$ R. Kiuchi, ${ }^{1}$ R. Kliemt, ${ }^{11}$ L. Koch,${ }^{30}$ O. B. Kolcu ${ }^{51 b, e}$ B. Kopf, ${ }^{4}$ M. Kuemmel, ${ }^{4}$ M. Kuessner, ${ }^{4}$ A. Kupsc, ${ }^{64}$ M. G. Kurth, ${ }^{1,52}$ W. Kühn, ${ }^{30}$ J. J. Lane, ${ }^{55}$ J. S. Lange ${ }^{30}{ }^{\text {P. Larin }},{ }^{15}$ L. Lavezzi,${ }^{63 a, 63 c}$ H. Leithoff, ${ }^{28}$ M. Lellmann, ${ }^{28}$ T. Lenz, ${ }^{28}$ C. Li ${ }^{39}$ C. H. Li ${ }^{33}$ Cheng Li,${ }^{60,48}$ D. M. Li ${ }^{68}$ F. Li, ${ }^{1,48}$ G. Li ${ }^{1}$ H. B. Li, ${ }^{1,52}$ H. J. Li, ${ }^{9}$ h J. L. Li, ${ }^{41}$ J. Q. Li, ${ }^{4} \mathrm{Ke} \mathrm{Li},{ }^{1}$ L. K. Li, ${ }^{1}$ Lei Li ${ }^{3}$ P.L. Li, ${ }^{60,48}$ P. R. Li, ${ }^{32}$ S. Y. Li ${ }^{50}$ W. D. Li, ${ }^{1,52}$ W. G. Li, ${ }^{1}$ X. H. Li ${ }^{60,48}$ X. L. Li ${ }^{41}$ Z. B. Li ${ }^{49}$ Z. Y. Li ${ }^{49}$ H. Liang, ${ }^{60,48}$ H. Liang, ${ }^{1,52}$ Y. F. Liang, ${ }^{45}$ Y. T. Liang, ${ }^{25}$ L. Z. Liao, ${ }^{1,52}$ J. Libby, ${ }^{21}$ C. X. Lin, ${ }^{49}$ B. Liu, ${ }^{42, g}$ B. J. Liu, ${ }^{1}$ C. X. Liu, ${ }^{1}$ D. Liu ${ }^{60,48}$ D. Y. Liu, ${ }^{42, g}$ F. H. Liu, ${ }^{44}$ Fang Liu, ${ }^{1}$ Feng Liu, ${ }^{6}$ H. B. Liu, ${ }^{13}$ H. M. Liu, ${ }^{1,52}$ Huanhuan Liu, ${ }^{1}$ Huihui Liu, ${ }_{17}$ J. B. Liu, ${ }^{60,48}$ J. Y. Liu, ${ }^{1,52}$ K. Liu, ${ }^{1}$ K. Y. Liu, ${ }^{34}$ Ke Liu, ${ }^{6}$ L. Liu, ${ }^{60,48}$ Q. Liu, ${ }^{52}$ S. B. Liu ${ }^{60,48}$ Shuai Liu, ${ }^{46}$ T. Liu ${ }^{1,52}$ X. Liu, ${ }^{32}$ Y. B. Liu, ${ }^{37}$ Z. A. Liu, ${ }^{1,48,52}$ Z. Q. Liu, ${ }^{41}$ Y. F. Long, ${ }^{38, k}$ X. C. Lou ${ }^{1,48,52}$ F. X. Lu, ${ }^{16}$ H. J. Lu, ${ }^{18}$ J. D. Lu, ${ }^{1,52}$ J. G. Lu, ${ }^{1,48}$ X. L. Lu, ${ }^{1}$ Y. Lu, ${ }^{1}$ Y. P. Lu, ${ }^{1,48}$ C. L. Luo, ${ }^{35}$ M. X. Luo, ${ }^{67}$ P. W. Luo, ${ }^{49}$ T. Luo, ${ }^{9, h}$ X. L. Luo, ${ }^{1,48}$ S. Lusso, ${ }^{63 c}$ X. R. Lyu ${ }^{52}$ F. C. Ma ${ }^{34}$ H. L. Ma, ${ }^{1}$ L. L. Ma ${ }^{41}$ M. M. Ma ${ }^{1,52}$ Q. M. Ma, ${ }^{1}$ R. Q. Ma, ${ }^{1,52}$ R. T. Ma ${ }^{52}$ X. N. Ma ${ }^{37}$ X. X. Ma,${ }^{1,52}$ X. Y. Ma,${ }^{1,48}$ Y. M. Ma ${ }^{41}$ F. E. Maas, ${ }^{15}$ M. Maggiora, ${ }^{63 a, 63 c}$ S. Maldaner, ${ }^{28}$ S. Malde, ${ }^{58}$ Q. A. Malik, ${ }^{62}$ A. Mangoni, ${ }^{23 b}$ Y. J. Mao, ${ }^{38, k}$ Z. P. Mao, ${ }^{1}$ S. Marcello, ${ }^{63 a, 63 c}$ Z. X. Meng, ${ }^{54}$ J. G. Messchendorp, ${ }^{31}$

G. Mezzadri, ${ }^{24 a}$ T. J. Min ${ }^{36}$ R. E. Mitchell, ${ }^{22}$ X. H. Mo, ${ }^{1,48,52}$ Y. J. Mo, ${ }^{6}$ N. Yu. Muchnoi, ${ }^{10, c}$ H. Muramatsu, ${ }^{56}$

S. Nakhoul, ${ }^{11, \mathrm{f}}$ Y. Nefedov, ${ }^{29}$ F. Nerling, ${ }^{11, \mathrm{f}}$ I. B. Nikolaev, ${ }^{10, \mathrm{c}}$ Z. Ning, ${ }^{1,48}$ S. Nisar, ${ }^{8, \mathrm{i}}$ S. L. Olsen, ${ }^{52}$ Q. Ouyang, ${ }^{1,48,52}$ S. Pacetti, ${ }^{23 b, 23 c}$ X. Pan, ${ }^{9, h}$ Y. Pan,${ }^{55}$ A. Pathak, ${ }^{1}$ P. Patteri, ${ }^{23 a}$ M. Pelizaeus, ${ }^{4}$ H. P. Peng, ${ }^{60,48}$ K. Peters,${ }^{11, \mathrm{f}}$ J. Pettersson, ${ }^{64}$ J. L. Ping ${ }^{35}$ R. G. Ping, ${ }^{1,52}$ A. Pitka, ${ }^{4}$ R. Poling, ${ }^{56}$ V. Prasad, ${ }^{60,48}$ H. Qi ${ }^{60,48}$ H. R. Qi ${ }^{50}$ M. Qi,${ }^{36}$ T. Y. Qi,${ }^{9}$ T. Y. Qi ${ }^{2}$

S. Qian, ${ }^{1,48}$ W.-B. Qian, ${ }^{52}$ Z. Qian, ${ }^{49}$ C. F. Qiao, ${ }^{52}$ L. Q. Qin, ${ }^{12}$ X. S. Qin, ${ }^{4}$ Z. H. Qin, ${ }^{1,48}$ J. F. Qiu, ${ }^{1}$ S. Q. Qu, ${ }^{37}$

K. H. Rashid, ${ }^{62}$ K. Ravindran, ${ }^{21}$ C. F. Redmer, ${ }^{28}$ A. Rivetti, ${ }^{63}$ V. Rodin, ${ }^{31}$ M. Rolo, ${ }^{63 \mathrm{c}}$ G. Rong, ${ }^{1,52}$ Ch. Rosner, ${ }^{15}$ M. Rump, ${ }^{57}$ A. Sarantsev, ${ }^{29, d}$ Y. Schelhaas, ${ }^{28}$ C. Schnier, ${ }^{4}$ K. Schoenning, ${ }^{64}$ M. Scodeggio, ${ }^{24 a}$ D. C. Shan,${ }^{46}$ W. Shan, ${ }^{19}$ X. Y. Shan,${ }^{60,48}$ M. Shao, ${ }^{60,48}$ C. P. Shen, ${ }^{9}$ P. X. Shen, ${ }^{37}$ X. Y. Shen, ${ }^{1,52}$ H. C. Shi, ${ }^{60,48}$ R. S. Shi, ${ }^{1,52}$ X. Shi, ${ }^{1,48}$ X. D. Shi, ${ }^{60,48}$ J. J. Song, ${ }^{41}$ Q. Q. Song, ${ }^{60,48}$ W. M. Song, ${ }^{27,1}$ Y.X. Song, ${ }^{38, k}$ S. Sosio, ${ }^{63 a, 63 c}$ S. Spataro, ${ }^{63 a, 63 c}$ F. F. Sui, ${ }^{41}$ G. X. Sun, ${ }^{1}$ J. F. Sun, ${ }^{16}$ L. Sun ${ }^{65}$ S. S. Sun, ${ }^{1,52}$ T. Sun, ${ }^{1,52}$ W. Y. Sun, ${ }^{35}$ X. Sun, ${ }^{20,1}$ Y. J. Sun,${ }^{6,48}$ Y. K. Sun ${ }^{60,48}$ Y. Z. Sun, ${ }^{1}$ Z. T. Sun, ${ }^{1}$ Y. H. Tan ${ }^{65}$ Y. X. Tan, ${ }^{60,48}$ C. J. Tang, ${ }^{45}$ G. Y. Tang, ${ }^{1}$ J. Tang, ${ }^{49}$ V. Thoren, ${ }^{64}$ B. Tsednee, ${ }^{26}$ I. Uman, ${ }^{51 \mathrm{~d}}$ B. Wang, ${ }^{1}$ B. L. Wang, ${ }^{52}$ C. W. Wang, ${ }^{36}$ D. Y. Wang, ${ }^{38, k}$ H. P. Wang, ${ }^{1,52}$ K. Wang, ${ }^{1,48}$ L. L. Wang, ${ }^{1}$ M. Wang, ${ }^{41}$ M. Z. Wang, ${ }^{38, k}$ Meng Wang, ${ }^{1,52}$ W. H. Wang, ${ }^{65}$ W. P. Wang, ${ }^{60,48}$ X. Wang, ${ }^{38, k}$ X. F. Wang, ${ }^{32}$ X. L. Wang, ${ }^{9, \mathrm{~h}}$ Y. Wang, ${ }^{49}$ Y. Wang, ${ }^{60,48}$ Y. D. Wang, ${ }^{15}$ Y. F. Wang, ${ }^{1,48,52}$ Y. Q. Wang, ${ }^{1}$ Z. Wang, ${ }^{1,48}$ Z. Y. Wang, ${ }^{1}$ Ziyi Wang, ${ }^{52}$ Zongyuan Wang, ${ }^{1,52}$ D. H. Wei, ${ }^{12}$ P. Weidenkaff, ${ }^{28}$ F. Weidner, ${ }^{57}$ S. P. Wen, ${ }^{1}$ D. J. White ${ }^{55}$ U. Wiedner, ${ }^{4}$ G. Wilkinson, ${ }^{58}$ M. Wolke, ${ }^{64}$ L. Wollenberg, ${ }^{4}$ J. F. Wu, ${ }^{1,52}$ L. H. Wu, ${ }^{1}$ L. J. Wu, ${ }^{1,52}$ X. Wu ${ }^{9, h}$ Z. Wu, ${ }^{1,48}$ L. Xia ${ }^{60,48}$ H. Xiao ${ }^{9, h}$ S. Y. Xiao, ${ }^{1}$ Y. J. Xiao, ${ }^{1,52}$ Z. J. Xiao, ${ }^{35}$ X. H. Xie, ${ }^{38, k}$ Y. G. Xie, ${ }^{1,48}$ Y. H. Xie, ${ }^{6}$ T. Y. Xing, ${ }^{1,52}$ X. A. Xiong, ${ }^{1,52}$ G. F. Xu, ${ }^{1}$ J. J. Xu, ${ }^{36}$ Q. J. Xu, ${ }^{14}$ W. Xu, ${ }^{1,52}$ X. P. Xu, ${ }^{46}$

F. Yan, ${ }^{9, h}$ L. Yan, ${ }^{63 a, 63 c}$ L. Yan, ${ }^{9, h}$ W. B. Yan, ${ }^{60,48}$ W. C. Yan, ${ }^{68}$ Xu Yan, ${ }^{46}$ H. J. Yang, ${ }^{42, g}$ H. X. Yang, ${ }^{1}$ L. Yang, ${ }^{65}$

R. X. Yang, ${ }^{6,48}$ S. L. Yang, ${ }^{1,52}$ Y. H. Yang, ${ }^{36}$ Y. X. Yang, ${ }^{12}$ Yifan Yang, ${ }^{1,52}$ Zhi Yang, ${ }^{25}$ M. Ye, ${ }^{1,48}$ M. H. Ye, J. H. Yin, ${ }^{1}$ Z. Y. You, ${ }^{49}$ B.X. Yu, ${ }^{1,48,52}$ C. X. Yu, ${ }^{37}$ G. Yu, ${ }^{1,52}$ J. S. Yu, ${ }^{20,1}$ T. Yu, ${ }^{61}$ C.Z. Yuan, ${ }^{1,52}$ W. Yuan, ${ }^{63 a, 63 c}$ X. Q. Yuan, ${ }^{38, k}$ Y. Yuan, ${ }^{1}$ Z. Y. Yuan, ${ }^{49}$ C. X. Yue, ${ }^{33}$ A. Yuncu, ${ }^{51 b, a}$ A. A. Zafar, ${ }^{62}$ Y. Zeng, ${ }^{20,1}$ B. X. Zhang, ${ }^{1}$ Guangyi Zhang, ${ }^{16}$ H. H. Zhang, ${ }^{49}$ H. Y. Zhang, ${ }^{1,48}$ J. L. Zhang, ${ }^{66}$ J. Q. Zhang, ${ }^{4}$ J. W. Zhang, ${ }^{1,48,52}$ J. Y. Zhang, ${ }^{1}$ J. Z. Zhang, ${ }^{1,52}$ 
Jianyu Zhang,, ${ }^{1,52}$ Jiawei Zhang, ${ }^{1,52}$ L. Zhang, ${ }^{1}$ Lei Zhang, ${ }^{36}$ S. Zhang, ${ }^{49}$ S. F. Zhang, ${ }^{36}$ T. J. Zhang, ${ }^{42, g}$ X. Y. Zhang, ${ }^{41}$ Y. Zhang, ${ }^{58}$ Y. H. Zhang, ${ }^{1,48}$ Y. T. Zhang, ${ }^{60,48}$ Yan Zhang, ${ }^{60,48}$ Yao Zhang, ${ }^{1}$ Yi Zhang, ${ }^{9, \mathrm{~h}}$ Z. H. Zhang, ${ }^{6}$ Z. Y. Zhang, ${ }^{65}$ G. Zhao, ${ }^{1}$ J. Zhao, ${ }^{33}$ J. Y. Zhao, ${ }^{1,52}$ J. Z. Zhao, ${ }^{1,48}$ Lei Zhao, ${ }^{60,48}$ Ling Zhao, ${ }^{1}$ M. G. Zhao, ${ }^{37}$ Q. Zhao, ${ }^{1}$ S. J. Zhao, ${ }^{68}$ Y. B. Zhao, ${ }^{1,48}$ Y. X. Zhao, ${ }^{25}$ Z. G. Zhao, ${ }^{60,48}$ A. Zhemchugov, ${ }^{29, b}$ B. Zheng, ${ }^{61}$ J. P. Zheng, ${ }^{1,48}$ Y. Zheng, ${ }^{38, k}$ Y. H. Zheng, ${ }^{52}$ B. Zhong, ${ }^{35}$ C. Zhong, ${ }^{61}$ L. P. Zhou, ${ }^{1,52}$ Q. Zhou, ${ }^{1,52}$ X. Zhou, ${ }^{65}$ X. K. Zhou, ${ }^{52}$ X. R. Zhou, ${ }^{60,48}$ A. N. Zhu, ${ }^{1,52}$ J. Zhu, ${ }^{37}$ K. Zhu, ${ }^{1}$ K. J. Zhu, ${ }^{1,48,52}$ S. H. Zhu, ${ }^{59}$ W. J. Zhu, ${ }^{37}$ X. L. Zhu, ${ }^{50}$ Y. C. Zhu, ${ }^{60,48}$ Z. A. Zhu, ${ }^{1,52}$ B. S. Zou, ${ }^{1}$ and J. H. Zou ${ }^{1}$

\title{
(BESIII Collaboration)
}

\author{
${ }^{1}$ Institute of High Energy Physics, Beijing 100049, People's Republic of China \\ ${ }^{2}$ Beihang University, Beijing 100191, People's Republic of China \\ ${ }^{3}$ Beijing Institute of Petrochemical Technology, Beijing 102617, People's Republic of China \\ ${ }^{4}$ Bochum Ruhr-University, D-44780 Bochum, Germany \\ ${ }^{5}$ Carnegie Mellon University, Pittsburgh, Pennsylvania 15213, USA \\ ${ }^{6}$ Central China Normal University, Wuhan 430079, People's Republic of China \\ ${ }^{7}$ China Center of Advanced Science and Technology, Beijing 100190, People's Republic of China \\ ${ }^{8}$ COMSATS University Islamabad, Lahore Campus, Defence Road, Off Raiwind Road, \\ 54000 Lahore, Pakistan \\ ${ }^{9}$ Fudan University, Shanghai 200443, People's Republic of China \\ ${ }^{10}$ G.I. Budker Institute of Nuclear Physics SB RAS (BINP), Novosibirsk 630090, Russia \\ ${ }^{11}$ GSI Helmholtzcentre for Heavy Ion Research GmbH, D-64291 Darmstadt, Germany \\ ${ }^{12}$ Guangxi Normal University, Guilin 541004, People's Republic of China \\ ${ }^{13}$ Guangxi University, Nanning 530004, People's Republic of China \\ ${ }^{14}$ Hangzhou Normal University, Hangzhou 310036, People's Republic of China \\ ${ }^{15}$ Helmholtz Institute Mainz, Johann-Joachim-Becher-Weg 45, D-55099 Mainz, Germany \\ ${ }^{16}$ Henan Normal University, Xinxiang 453007, People's Republic of China \\ ${ }^{17}$ Henan University of Science and Technology, Luoyang 471003, People's Republic of China \\ ${ }^{18}$ Huangshan College, Huangshan 245000, People's Republic of China \\ ${ }^{19}$ Hunan Normal University, Changsha 410081, People's Republic of China \\ ${ }^{20}$ Hunan University, Changsha 410082, People's Republic of China \\ ${ }^{21}$ Indian Institute of Technology Madras, Chennai 600036, India \\ ${ }^{22}$ Indiana University, Bloomington, Indiana 47405, USA \\ ${ }^{23}$ INFN Laboratori Nazionali di Frascati, I-00044, Frascati, Italy \\ ${ }^{23 \mathrm{~b}}$ INFN Sezione di Perugia, I-06100, Perugia, Italy \\ ${ }^{23 c}$ University of Perugia, I-06100, Perugia, Italy \\ ${ }^{24 a}$ INFN Sezione di Ferrara, I-44122, Ferrara, Italy \\ ${ }^{24 \mathrm{~b}}$ University of Ferrara, I-44122, Ferrara, Italy \\ ${ }^{25}$ Institute of Modern Physics, Lanzhou 730000, People's Republic of China \\ ${ }^{26}$ Institute of Physics and Technology, Peace Ave. 54B, Ulaanbaatar 13330, Mongolia \\ ${ }^{27}$ Jilin University, Changchun 130012, People's Republic of China \\ ${ }^{28}$ Johannes Gutenberg University of Mainz, Johann-Joachim-Becher-Weg 45, D-55099 Mainz, Germany \\ ${ }^{29}$ Joint Institute for Nuclear Research, 141980 Dubna, Moscow region, Russia \\ ${ }^{30}$ Justus-Liebig-Universitaet Giessen, II. Physikalisches Institut, Heinrich-Buff-Ring 16, \\ D-35392 Giessen, Germany \\ ${ }^{31}$ KVI-CART, University of Groningen, NL-9747 AA Groningen, Netherlands \\ ${ }^{32}$ Lanzhou University, Lanzhou 730000, People's Republic of China \\ ${ }^{33}$ Liaoning Normal University, Dalian 116029, People's Republic of China \\ ${ }^{34}$ Liaoning University, Shenyang 110036, People's Republic of China \\ ${ }^{35}$ Nanjing Normal University, Nanjing 210023, People's Republic of China \\ ${ }^{36}$ Nanjing University, Nanjing 210093, People's Republic of China \\ ${ }^{37}$ Nankai University, Tianjin 300071, People's Republic of China \\ ${ }^{38}$ Peking University, Beijing 100871, People's Republic of China \\ ${ }^{39}$ Qufu Normal University, Qufu 273165, People's Republic of China \\ ${ }^{40}$ Shandong Normal University, Jinan 250014, People's Republic of China \\ ${ }^{41}$ Shandong University, Jinan 250100, People's Republic of China \\ ${ }^{42}$ Shanghai Jiao Tong University, Shanghai 200240, People's Republic of China \\ ${ }^{43}$ Shanxi Normal University, Linfen 041004, People's Republic of China \\ ${ }^{44}$ Shanxi University, Taiyuan 030006, People's Republic of China \\ ${ }^{45}$ Sichuan University, Chengdu 610064, People's Republic of China
}




\author{
${ }^{46}$ Soochow University, Suzhou 215006, People's Republic of China \\ ${ }^{47}$ Southeast University, Nanjing 211100, People's Republic of China \\ ${ }^{48}$ State Key Laboratory of Particle Detection and Electronics, \\ Beijing 100049, Hefei 230026, People's Republic of China \\ ${ }^{49}$ Sun Yat-Sen University, Guangzhou 510275, People's Republic of China \\ ${ }^{50}$ Tsinghua University, Beijing 100084, People's Republic of China \\ ${ }^{51 a}$ Ankara University, 06100 Tandogan, Ankara, Turkey \\ ${ }^{51 \mathrm{~b}}$ Istanbul Bilgi University, 34060 Eyup, Istanbul, Turkey \\ ${ }^{51 \mathrm{c}}$ Uludag University, 16059 Bursa, Turkey \\ ${ }^{51 \mathrm{~d}}$ Near East University, Nicosia, North Cyprus, Mersin 10, Turkey \\ ${ }^{52}$ University of Chinese Academy of Sciences, Beijing 100049, People's Republic of China \\ ${ }^{53}$ University of Hawaii, Honolulu, Hawaii 96822, USA \\ ${ }^{54}$ University of Jinan, Jinan 250022, People's Republic of China \\ ${ }^{55}$ University of Manchester, Oxford Road, Manchester, M13 9PL, United Kingdom \\ ${ }^{56}$ University of Minnesota, Minneapolis, Minnesota 55455, USA \\ ${ }^{57}$ University of Muenster, Wilhelm-Klemm-Str. 9, 48149 Muenster, Germany \\ ${ }^{58}$ University of Oxford, Keble Rd, Oxford, OX13RH, United Kingdom \\ ${ }^{59}$ University of Science and Technology Liaoning, Anshan 114051, People's Republic of China \\ ${ }^{60}$ University of Science and Technology of China, Hefei 230026, People's Republic of China \\ ${ }^{61}$ University of South China, Hengyang 421001, People's Republic of China \\ ${ }^{62}$ University of the Punjab, Lahore-54590, Pakistan \\ ${ }^{63 a}$ University of Turin, I-10125, Turin, Italy \\ ${ }^{63 \mathrm{~b}}$ University of Eastern Piedmont, I-15121, Alessandria, Italy

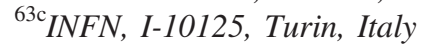 \\ ${ }^{64}$ Uppsala University, Box 516, SE-75120 Uppsala, Sweden \\ ${ }^{65}$ Wuhan University, Wuhan 430072, People's Republic of China \\ ${ }^{66}$ Xinyang Normal University, Xinyang 464000, People's Republic of China \\ ${ }^{67}$ Zhejiang University, Hangzhou 310027, People's Republic of China \\ ${ }^{68}$ Zhengzhou University, Zhengzhou 450001, People's Republic of China
}

(Received 21 July 2020; accepted 8 September 2020; published 25 September 2020)

Based on $e^{+} e^{-}$collision data sample corresponding to an integrated luminosity of $2.93 \mathrm{fb}^{-1}$ taken at a center-of-mass energy of $3.773 \mathrm{GeV}$ by the BESIII detector, we report the measurements of the absolute branching fractions of $D^{0} \rightarrow K^{+} K^{-} \pi^{0} \pi^{0}, D^{0} \rightarrow K_{S}^{0} K_{S}^{0} \pi^{+} \pi^{-}, D^{0} \rightarrow K_{S}^{0} K^{-} \pi^{+} \pi^{0}, D^{0} \rightarrow K_{S}^{0} K^{+} \pi^{-} \pi^{0}$, $D^{+} \rightarrow K^{+} K^{-} \pi^{+} \pi^{0}, D^{+} \rightarrow K_{S}^{0} K^{+} \pi^{0} \pi^{0}, D^{+} \rightarrow K_{S}^{0} K^{-} \pi^{+} \pi^{+}, D^{+} \rightarrow K_{S}^{0} K^{+} \pi^{+} \pi^{-}$, and $D^{+} \rightarrow K_{S}^{0} K_{S}^{0} \pi^{+} \pi^{0}$. The decays $D^{0} \rightarrow K^{+} K^{-} \pi^{0} \pi^{0}, D^{0} \rightarrow K_{S}^{0} K^{-} \pi^{+} \pi^{0}, D^{0} \rightarrow K_{S}^{0} K^{+} \pi^{-} \pi^{0}, D^{+} \rightarrow K_{S}^{0} K_{S}^{0} \pi^{+} \pi^{0}$, and $D^{+} \rightarrow$ $K_{S}^{0} K^{+} \pi^{0} \pi^{0}$ are observed for the first time. The branching fractions of the decays $D^{0} \rightarrow K_{S}^{0} K_{S}^{0} \pi^{+} \pi^{-}$, $D^{+} \rightarrow K^{+} K^{-} \pi^{+} \pi^{0}, D^{+} \rightarrow K_{S}^{0} K^{-} \pi^{+} \pi^{+}$, and $D^{+} \rightarrow K_{S}^{0} K^{+} \pi^{+} \pi^{-}$are measured with improved precision compared to the world-average values.

DOI: $10.1103 /$ PhysRevD.102.052006

${ }^{\mathrm{a}}$ Also at Bogazici University, 34342 Istanbul, Turkey.

${ }^{\mathrm{b}}$ Also at the Moscow Institute of Physics and Technology, Moscow 141700, Russia.

${ }^{\mathrm{c}}$ Also at the Novosibirsk State University, Novosibirsk, 630090, Russia.

dAlso at the NRC "Kurchatov Institute", PNPI, 188300, Gatchina, Russia.

${ }^{\mathrm{e} A}$ Also at Istanbul Arel University, 34295 Istanbul, Turkey.

${ }^{\mathrm{f}}$ Also at Goethe University Frankfurt, 60323 Frankfurt am Main, Germany.

${ }^{g}$ Also at Key Laboratory for Particle Physics, Astrophysics and Cosmology, Ministry of Education; Shanghai Key Laboratory for Particle Physics and Cosmology; Institute of Nuclear and Particle Physics, Shanghai 200240, People's Republic of China.

${ }^{\mathrm{h}}$ Also at Key Laboratory of Nuclear Physics and Ion-beam Application (MOE) and Institute of Modern Physics, Fudan University, Shanghai 200443, People's Republic of China.

${ }^{i}$ Also at Harvard University, Department of Physics, Cambridge, Massachusetts 02138, USA.

${ }^{\mathrm{j}}$ Currently at: Institute of Physics and Technology, Peace Ave. 54B, Ulaanbaatar 13330, Mongolia.

${ }^{k}$ Also at State Key Laboratory of Nuclear Physics and Technology, Peking University, Beijing 100871, People's Republic of China.

${ }^{1}$ Also at School of Physics and Electronics, Hunan University, Changsha 410082, China.

Published by the American Physical Society under the terms of the Creative Commons Attribution 4.0 International license. Further distribution of this work must maintain attribution to the author(s) and the published article's title, journal citation, and DOI. Funded by SCOAP. 


\section{INTRODUCTION}

Multibody hadronic $D^{0(+)}$ decays provide an ideal laboratory to study strong and weak interactions. Amplitude analyses of these decays offer comprehensive information of quasi-two-body $D^{0(+)}$ decays, which are important to explore $D \bar{D}^{0}$ mixing, charge conjugationparity $(C P)$ violation, and quark $\mathrm{SU}(3)$-flavor asymmetry breaking phenomenon [1-5]. In particular, for the search of $C P$ violation, it is important to understand the intermediate structures for the singly Cabibbo-suppressed decays of $D^{0(+)} \rightarrow K \bar{K} \pi \pi[6-8]$.

Current measurements of the $D^{0(+)} \rightarrow K \bar{K} \pi \pi$ decays containing $K_{S}^{0}$ or $\pi^{0}$ are limited [9]. The branching fractions (BFs) of $D^{0} \rightarrow K_{S}^{0} K_{S}^{0} \pi^{+} \pi^{-}$[10,11], $D^{+} \rightarrow K_{S}^{0} K^{-} \pi^{+} \pi^{+}$ [12], $D^{+} \rightarrow K_{S}^{0} K^{+} \pi^{+} \pi^{-}$[12], and $D^{+} \rightarrow K^{+} K^{-} \pi^{+} \pi^{0}$ [13] were only determined relative to some well-known decays or via topological normalization, with poor precision. This paper presents the first direct measurements of the absolute BFs for the decays $D^{0} \rightarrow K^{+} K^{-} \pi^{0} \pi^{0}, D^{0} \rightarrow K_{S}^{0} K_{S}^{0} \pi^{+} \pi^{-}$, $D^{0} \rightarrow K_{S}^{0} K^{-} \pi^{+} \pi^{0}, D^{0} \rightarrow K_{S}^{0} K^{+} \pi^{-} \pi^{0}, D^{+} \rightarrow K^{+} K^{-} \pi^{+} \pi^{0}$, $D^{+} \rightarrow K_{S}^{0} K^{+} \pi^{0} \pi^{0}, D^{+} \rightarrow K_{S}^{0} K^{-} \pi^{+} \pi^{+}, D^{+} \rightarrow K_{S}^{0} K^{+} \pi^{+} \pi^{-}$, and $D^{+} \rightarrow K_{S}^{0} K_{S}^{0} \pi^{+} \pi^{0}$. The $D^{0} \rightarrow K_{S}^{0} K_{S}^{0} \pi^{0} \pi^{0}$ decay is not included since it suffers from poor statistics and high background. Throughout this paper, charge conjugate processes are implied. An $e^{+} e^{-}$collision data sample corresponding to an integrated luminosity of $2.93 \mathrm{fb}^{-1}$ [14] collected at a center-of-mass energy of $\sqrt{s}=3.773 \mathrm{GeV}$ with the BESIII detector is used to perform this analysis.

\section{BESIII DETECTOR AND MONTE CARLO SIMULATION}

The BESIII detector is a magnetic spectrometer [15] located at the Beijing Electron Positron Collider (BEPCII) [16]. The cylindrical core of the BESIII detector consists of a helium-based multilayer drift chamber (MDC), a plastic scintillator time-of-flight system (TOF), and a CsI(Tl) electromagnetic calorimeter (EMC), which are all enclosed in a superconducting solenoidal magnet providing an $1.0 \mathrm{~T}$ magnetic field. The solenoid is supported by an octagonal flux-return yoke with resistive plate counter muon identifier modules interleaved with steel. The acceptance of charged particles and photons is $93 \%$ over $4 \pi$ solid angle. The charged-particle momentum resolution at $1 \mathrm{GeV} / c$ is $0.5 \%$, and the $d E / d x$ resolution is $6 \%$ for the electrons from Bhabha scattering. The EMC measures photon energies with a resolution of $2.5 \%(5 \%)$ at $1 \mathrm{GeV}$ in the barrel (end cap) region. The time resolution of the TOF barrel part is $68 \mathrm{ps}$, while that of the end cap part is $110 \mathrm{ps}$.

Simulated samples produced with the GEANT4-based [17] Monte Carlo (MC) package including the geometric description of the BESIII detector and the detector response, are used to determine the detection efficiency and to estimate the backgrounds. The simulation includes the beam-energy spread and initial-state radiation (ISR) in the $e^{+} e^{-}$annihilations modeled with the generator KKMC [18]. The inclusive MC samples consist of the production of $D \bar{D}$ pairs with consideration of quantum coherence for all neutral $D$ modes, the non- $D \bar{D}$ decays of the $\psi(3770)$, the ISR production of the $J / \psi$ and $\psi(3686)$ states, and the continuum processes. The known decay modes are modeled with EVTGEN [19] using the BFs taken from the Particle Data Group (PDG) [9], and the remaining unknown decays from the charmonium states are modeled with LUNDCHARM [20]. The final-state radiations from charged final-state particles are incorporated with the PHOTOS package [21].

\section{MEASUREMENT METHOD}

The $D^{0} \bar{D}^{0}$ or $D^{+} D^{-}$pair is produced without an additional hadron in $e^{+} e^{-}$annihilations at $\sqrt{s}=3.773 \mathrm{GeV}$. This process offers a clean environment to measure the BFs of the hadronic $D$ decay with the double-tag (DT) method. The single-tag (ST) candidate events are selected by reconstructing a $\bar{D}^{0}$ or $D^{-}$in the following hadronic final states: $\bar{D}^{0} \rightarrow K^{+} \pi^{-}, \quad K^{+} \pi^{-} \pi^{0}$, and $K^{+} \pi^{-} \pi^{-} \pi^{+}$, and $D^{-} \rightarrow K^{+} \pi^{-} \pi^{-}, K_{S}^{0} \pi^{-}, K^{+} \pi^{-} \pi^{-} \pi^{0}, K_{S}^{0} \pi^{-} \pi^{0}, K_{S}^{0} \pi^{+} \pi^{-} \pi^{-}$, and $K^{+} K^{-} \pi^{-}$. The event in which a signal candidate is selected in the presence of an ST $\bar{D}$ meson, is called a DT event. The BF of the signal decay is determined by

$$
\mathcal{B}_{\text {sig }}=N_{\mathrm{DT}}^{\text {net }} /\left(N_{\mathrm{ST}}^{\mathrm{tot}} \cdot \epsilon_{\mathrm{sig}}\right),
$$

where $N_{\mathrm{ST}}^{\mathrm{tot}}=\sum_{i} N_{\mathrm{ST}}^{i}$ and $N_{\mathrm{DT}}^{\text {net }}$ are the total yields of the ST and DT candidates in data, respectively. $N_{\mathrm{ST}}^{i}$ is the ST yield for the tag mode $i$. For the signal decays involving $K_{S}^{0}$ meson(s) in the final states, $N_{\mathrm{DT}}^{\text {net }}$ is the net DT yields after removing the peaking background from the corresponding non- $K_{S}^{0}$ decays. For the other signal decays, the variable corresponds to the fitted DT yields as described later. Here, $\epsilon_{\text {sig }}$ is the efficiency of detecting the signal $D$ decay, averaged over the tag mode $i$, which is given by:

$$
\epsilon_{\mathrm{Sig}}=\sum_{i}\left(N_{\mathrm{ST}}^{i} \cdot \epsilon_{\mathrm{DT}}^{i} / \epsilon_{\mathrm{ST}}^{i}\right) / N_{\mathrm{ST}}^{\mathrm{tot}},
$$

where $\epsilon_{\mathrm{ST}}^{i}$ and $\epsilon_{\mathrm{DT}}^{i}$ are the efficiencies of detecting ST and DT candidates in the tag mode $i$, respectively. For $D^{0}$ decay, the signal efficiency has been corrected by a factor considering the Cabibbo-favored and doubly Cabibbosuppressed contributions of the tag sides, as shown in Sec. VIII.

\section{EVENT SELECTION}

The selection criteria of $K^{ \pm}, \pi^{ \pm}, K_{S}^{0}$, and $\pi^{0}$ are the same as those used in the analyses presented in Refs. [22-31]. All charged tracks, except those from $K_{S}^{0}$ decays, are required 
to have a polar angle $\theta$ with respect to the beam direction within the MDC acceptance $|\cos \theta|<0.93$, and a distance of closest approach to the interaction point (IP) within $10 \mathrm{~cm}$ along the beam direction and within $1 \mathrm{~cm}$ in the plane transverse to the beam direction. Particle identification (PID) for charged pions, kaons, and protons is performed by exploiting TOF information and the specific ionization energy loss $d E / d x$ measured by the MDC. The confidence levels for pion and kaon hypotheses $\left(C L_{\pi}\right.$ and $C L_{K}$ ) are calculated. Kaon and pion candidates are required to satisfy $C L_{K}>C L_{\pi}$ and $C L_{\pi}>C L_{K}$, respectively.

The $K_{S}^{0}$ candidates are reconstructed from two oppositely charged tracks to which no PID criteria are applied and which masses are assumed to be that of pions. The charged tracks from the $K_{S}^{0}$ candidate must satisfy $|\cos \theta|<0.93$. In addition, due to the long lifetime of the $K_{S}^{0}$ meson, there is a less stringent criterion on the distance of closest approach to the IP in the beam direction of less than $20 \mathrm{~cm}$ and no requirement on the distance of closest approach in the plane transverse to the beam direction. Furthermore, the $\pi^{+} \pi^{-}$ pairs are constrained to originate from a common vertex and their invariant mass is required to be within $(0.486,0.510) \mathrm{GeV} / c^{2}$, which corresponds to about three times the fitted resolution around the nominal $K_{S}^{0}$ mass. The decay length of the $K_{S}^{0}$ candidate is required to be larger than two standard deviations of the vertex resolution away from the IP.

The $\pi^{0}$ candidate is reconstructed via its $\gamma \gamma$ decay. The photon candidates are selected using the information from the EMC shower. It is required that each EMC shower starts within $700 \mathrm{~ns}$ of the event start time and its energy is greater than 25 (50) $\mathrm{MeV}$ in the barrel (end cap) region of the EMC [15]. The energy deposited in the nearby TOF counters is included to improve the reconstruction efficiency and energy resolution. The opening angle between the candidate shower and the nearest charged track must be greater than $10^{\circ}$. The $\gamma \gamma$ pair is taken as a $\pi^{0}$ candidate if its invariant mass is within $(0.115,0.150) \mathrm{GeV} / c^{2}$. To improve the resolution, a kinematic fit constraining the $\gamma \gamma$ invariant mass to the $\pi^{0}$ nominal mass [9] is imposed on the selected photon pair.

\section{YIELDS OF ST $\bar{D}$ MESONS}

To select $\bar{D}^{0} \rightarrow K^{+} \pi^{-}$candidates, the backgrounds from cosmic rays and Bhabha events are rejected by using the same requirements described in Ref. [32]. In the selection of $\bar{D}^{0} \rightarrow K^{+} \pi^{-} \pi^{-} \pi^{+}$candidates, the $\bar{D}^{0} \rightarrow K_{S}^{0} K^{ \pm} \pi^{\mp}$ decays are suppressed by requiring the mass of all $\pi^{+} \pi^{-}$ pairs to be outside $(0.478,0.518) \mathrm{GeV} / c^{2}$.

The tagged $\bar{D}$ mesons are identified using two variables, namely the energy difference

$$
\Delta E_{\mathrm{tag}} \equiv E_{\mathrm{tag}}-E_{\mathrm{b}},
$$

and the beam-constrained mass

$$
M_{\mathrm{BC}}^{\mathrm{tag}} \equiv \sqrt{E_{\mathrm{b}}^{2}-\left|\vec{p}_{\mathrm{tag}}\right|^{2}} .
$$

Here, $E_{\mathrm{b}}$ is the beam energy, $\vec{p}_{\text {tag }}$ and $E_{\text {tag }}$ are the momentum and energy of the $\bar{D}$ candidate in the rest frame of $e^{+} e^{-}$system, respectively. For each tag mode, if there are multiple candidates in an event, only the one with the smallest $\left|\Delta E_{\text {tag }}\right|$ is kept. The tagged $\bar{D}$ candidates are required to satisfy $\Delta E_{\mathrm{tag}} \in(-55,40) \mathrm{MeV}$ for the tag modes containing $\pi^{0}$ in the final states and $\Delta E_{\text {tag }} \in$ $(-25,25) \mathrm{MeV}$ for the other tag modes, thereby taking into account the different resolutions.

To extract the yields of ST $\bar{D}$ mesons for individual tag modes, binned-maximum likelihood fits are performed on the corresponding $M_{\mathrm{BC}}^{\mathrm{tag}}$ distributions of the accepted ST candidates following Refs. [22-28]. In the fits, the $\bar{D}$ signal is modeled by an MC-simulated shape convolved with a double-Gaussian function describing the resolution difference between data and MC simulation. The combinatorial background shape is described by an ARGUS function [33] defined as $c_{f}\left(f ; E_{\mathrm{end}}, \xi_{f}\right)=A_{f} \cdot f \cdot \sqrt{1-\left(f^{2} / E_{\mathrm{end}}^{2}\right)}$. $\exp \left[\xi_{f}\left(1-f^{2} / E_{\text {end }}^{2}\right)\right]$, where $f$ denotes $M_{\mathrm{BC}}^{\mathrm{tag}}, E_{\text {end }}$ is an endpoint fixed at $1.8865 \mathrm{GeV}, A_{f}$ is a normalization factor, and $\xi_{f}$ is a free parameter. The resulting fits to the $M_{\mathrm{BC}}$ distributions for each mode are shown in Fig. 1. The total yields of the ST $\bar{D}^{0}$ and $D^{-}$mesons in data are $2327839 \pm$ 1860 and $1558159 \pm 2113$, respectively, where the uncertainties are statistical only. For $D^{-} \rightarrow K_{S}^{0} \pi^{-}$, $D^{-} \rightarrow K_{S}^{0} \pi^{-} \pi^{0}$, and $D^{-} \rightarrow K_{S}^{0} \pi^{+} \pi^{-} \pi^{-}$, small non- $K_{S}^{0}$ contributions $(<0.5 \%$ for each mode) can be used as tags and retained in the ST yields. The $D^{-} \rightarrow K_{S}^{0} K_{S}^{0} \pi^{-}$decays are kept as $D^{-} \rightarrow K_{S}^{0} \pi^{+} \pi^{-} \pi^{-} \mathrm{ST}$ candidates $(\sim 4 \%)$. Especially,
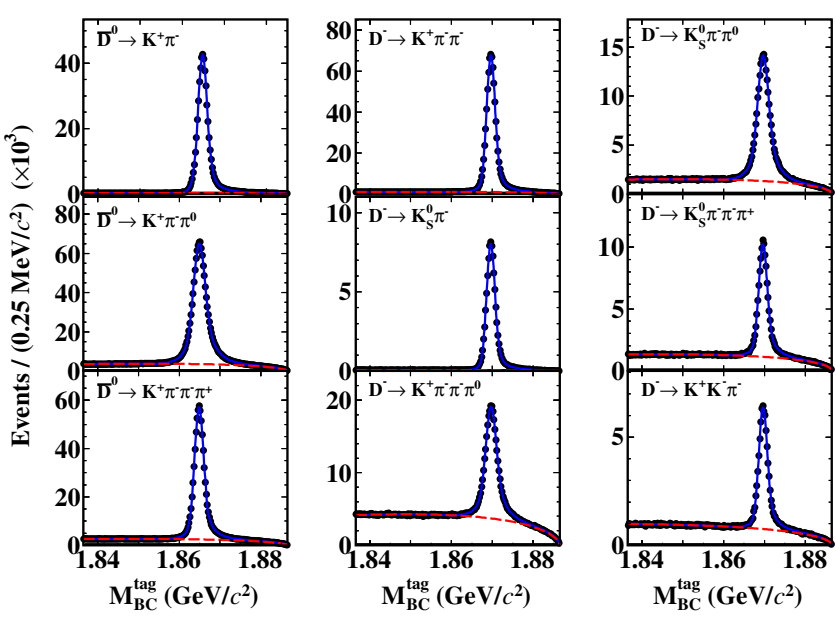

FIG. 1. Fits to the $M_{\mathrm{BC}}$ distributions of the ST $\bar{D}^{0}$ (left column) and $D^{-}$(middle and right columns) candidates, where the points with error bars are data, the blue solid and red dashed curves are the fit results and the fitted backgrounds, respectively. 
TABLE I. Requirements of $\Delta E_{\text {sig }}$, the fitted DT yields in the $K_{S}^{0}$ signal region $\left(N_{\mathrm{DT}}^{\mathrm{fit}}\right)$, the fitted DT yields in the $K_{S}^{0}$ sideband region $\left(N_{K_{S}^{0}}^{\text {sid }}\right)$, the net DT yields $\left(N_{\mathrm{DT}}^{\text {net }}\right)$, the signal efficiencies $\left(\epsilon_{\text {sig }}\right)$, and the obtained BFs $\left(\mathcal{B}_{\text {sig }}\right)$ for various signal decays as well as comparisons with the world-average BFs $\left(\mathcal{B}_{\mathrm{PDG}}\right)$. The first and second uncertainties for $\mathcal{B}_{\text {sig }}$ are statistical and systematic, respectively, while the uncertainties for $N_{\mathrm{DT}}^{\text {net }}$ and $\epsilon_{\text {sig }}$ are statistical only. The world-average BF of $D^{+} \rightarrow K^{+} K^{-} \pi^{+} \pi^{0}$ is obtained by summing over the contributions of $D^{+} \rightarrow \phi\left(\rightarrow K^{+} K^{-}\right) \pi^{+} \pi^{0}$ and $\left.D^{+} \rightarrow K^{+} K^{-} \pi^{+} \pi^{0}\right|_{\text {non- } \phi}$.

\begin{tabular}{lccccccc}
\hline \hline Signal mode & $\Delta E_{\text {sig }}$ & $N_{\mathrm{DT}}^{\text {fit }}$ & $N_{K_{S}^{0}}^{\text {sid }}$ & $N_{\mathrm{DT}}^{\text {net }}$ & $\epsilon_{\text {sig }}(\%)$ & $\mathcal{B}_{\text {sig }}\left(\times 10^{-3}\right)$ & $\mathcal{B}_{\mathrm{PDG}}\left(\times 10^{-3}\right)$ \\
\hline$D^{0} \rightarrow K^{+} K^{-} \pi^{0} \pi^{0}$ & $(-59,40)$ & $132.1 \pm 13.9$ & $\ldots$ & $132.1 \pm 13.9$ & $8.20 \pm 0.07$ & $0.69 \pm 0.07 \pm 0.04$ & $\ldots$ \\
$D^{0} \rightarrow K_{S}^{0} K_{S}^{0} \pi^{+} \pi^{-}$ & $(-22,22)$ & $82.1 \pm 9.7$ & $37.8 \pm 7.5$ & $63.2 \pm 10.4$ & $5.14 \pm 0.04$ & $0.53 \pm 0.09 \pm 0.03$ & $1.22 \pm 0.23$ \\
$D^{0} \rightarrow K_{S}^{0} K^{-} \pi^{+} \pi^{0}$ & $(-43,32)$ & $278.8 \pm 18.8$ & $166.1 \pm 15.1$ & $195.8 \pm 20.3$ & $6.38 \pm 0.06$ & $1.32 \pm 0.14 \pm 0.07$ & $\ldots$ \\
$D^{0} \rightarrow K_{S}^{0} K^{+} \pi^{-} \pi^{0}$ & $(-44,33)$ & $124.0 \pm 12.8$ & $9.5_{-3.1}^{+3.7}$ & $119.3 \pm 12.9$ & $7.94 \pm 0.06$ & $0.65 \pm 0.07 \pm 0.02$ & $\ldots$ \\
$D^{+} \rightarrow K^{+} K^{-} \pi^{+} \pi^{0}$ & $(-39,30)$ & $1311.7 \pm 40.4$ & $\ldots$ & $1311.7 \pm 40.4$ & $12.72 \pm 0.08$ & $6.62 \pm 0.20 \pm 0.25$ & $26_{-8}^{+9}$ \\
$D^{+} \rightarrow K_{S}^{0} K^{+} \pi^{0} \pi^{0}$ & $(-61,44)$ & $35.9 \pm 7.1$ & $3.8_{-2.0}^{+2.8}$ & $34.0 \pm 7.2$ & $3.77 \pm 0.02$ & $0.58 \pm 0.12 \pm 0.04$ & $\ldots$ \\
$D^{+} \rightarrow K_{S}^{0} K^{-} \pi^{+} \pi^{+}$ & $(-22,21)$ & $505.0 \pm 24.5$ & $74.2 \pm 10.3$ & $467.9 \pm 25.0$ & $13.24 \pm 0.08$ & $2.27 \pm 0.12 \pm 0.06$ & $2.38 \pm 0.17$ \\
$D^{+} \rightarrow K_{S}^{0} K^{+} \pi^{+} \pi^{-}$ & $(-21,20)$ & $284.6 \pm 18.0$ & $15.3_{-4.2}^{+4.9}$ & $277.0 \pm 18.2$ & $9.39 \pm 0.06$ & $1.89 \pm 0.12 \pm 0.05$ & $1.74 \pm 0.18$ \\
$D^{+} \rightarrow K_{S}^{0} K_{S}^{0} \pi^{+} \pi^{0}$ & $(-46,37)$ & $101.1 \pm 11.3$ & $42.0 \pm 8.1$ & $80.1 \pm 12.0$ & $3.84 \pm 0.03$ & $1.34 \pm 0.20 \pm 0.06$ & $\ldots$ \\
\hline \hline
\end{tabular}

the $\bar{D}^{0} \rightarrow K_{S}^{0} K^{+} \pi^{-}$and $\bar{D}^{0} \rightarrow K_{S}^{0} K^{-} \pi^{+}$decays, which contribute $\sim 2.6 \%$ to the $\bar{D}^{0} \rightarrow K^{+} \pi^{-} \pi^{-} \pi^{+}$ST candidates, have been subtracted due to the strong-phase effects.

\section{YIELDS OF DT EVENTS}

In the recoiling sides against the tagged $\bar{D}$ candidates, the signal $D$ decays are selected by using the residual tracks that have not been used to reconstruct the tagged $\bar{D}$ candidates. To suppress the $K_{S}^{0}$ contribution in the individual mass spectra for the $D^{0} \rightarrow K^{+} K^{-} \pi^{0} \pi^{0}, D^{0} \rightarrow$ $K_{S}^{0} K_{S}^{0} \pi^{+} \pi^{-}$, and $D^{+} \rightarrow K_{S}^{0} K^{+} \pi^{+} \pi^{-}$decays, the $\pi^{+} \pi^{-}$ and $\pi^{0} \pi^{0}$ invariant masses are required to be outside $(0.468,0.528) \mathrm{GeV} / c^{2}$ and $(0.438,0.538) \mathrm{GeV} / c^{2}$, respectively. To suppress the background from $D^{0} \rightarrow K^{-} \pi^{+} \omega$ in the identification of the $D^{0} \rightarrow K_{S}^{0} K^{-} \pi^{+} \pi^{0}$ process, the $K_{S}^{0} \pi^{0}$ invariant mass is required to be outside $(0.742$, $0.822) \mathrm{GeV} / c^{2}$. These requirements correspond to at least five times the fitted mass resolution away from the fitted mean of the mass peak.

The signal $D$ mesons are identified using the energy difference $\Delta E_{\text {sig }}$ and the beam-constrained mass $M_{\mathrm{BC}}^{\text {sig }}$, which are calculated with Eqs. (3) and (4) by substituting "tag" with "sig". For each signal mode, if there are multiple candidates in an event, only the one with the smallest $\left|\Delta E_{\text {sig }}\right|$ is kept. The signal decays are required to satisfy the mode-dependent $\Delta E_{\text {sig }}$ requirements, as shown in the second column of Table I. To suppress incorrectly identified $D \bar{D}$ candidates, the opening angle between the tagged $\bar{D}$ and the signal $D$ is required to be greater than $160^{\circ}$, resulting in a loss of (2-6)\% of the signal and suppressing $(8-55) \%$ of the background.

Figure 2 shows the $M_{\mathrm{BC}}^{\mathrm{tag}}$ versus $M_{\mathrm{BC}}^{\mathrm{sig}}$ distribution of the accepted DT candidates in data. The signal events concentrate around $M_{\mathrm{BC}}^{\mathrm{tag}}=M_{\mathrm{BC}}^{\mathrm{sig}}=M_{D}$, where $M_{D}$ is the nominal $D$ mass [9]. The events with correctly reconstructed $D(\bar{D})$ and incorrectly reconstructed $\bar{D}(D)$, named BKGI, are spread along the lines around $M_{\mathrm{BC}}^{\mathrm{tag}}=M_{D}$ or $M_{\mathrm{BC}}^{\mathrm{sig}}=M_{D}$. The events smeared along the diagonal, named BKGII, are mainly from the $e^{+} e^{-} \rightarrow q \bar{q}$ processes. The events with uncorrelated and incorrectly reconstructed $D$ and $\bar{D}$, named BKGIII, disperse across the whole allowed kinematic region. Usually, the horizonal and vertical BKGI components are caused mainly due to particle misidentification(s), fake or missing $\pi^{0}(\mathrm{~s})$ in the signal and tag sides, respectively. For example, inclusive MC studies show that the largest source of the horizonal

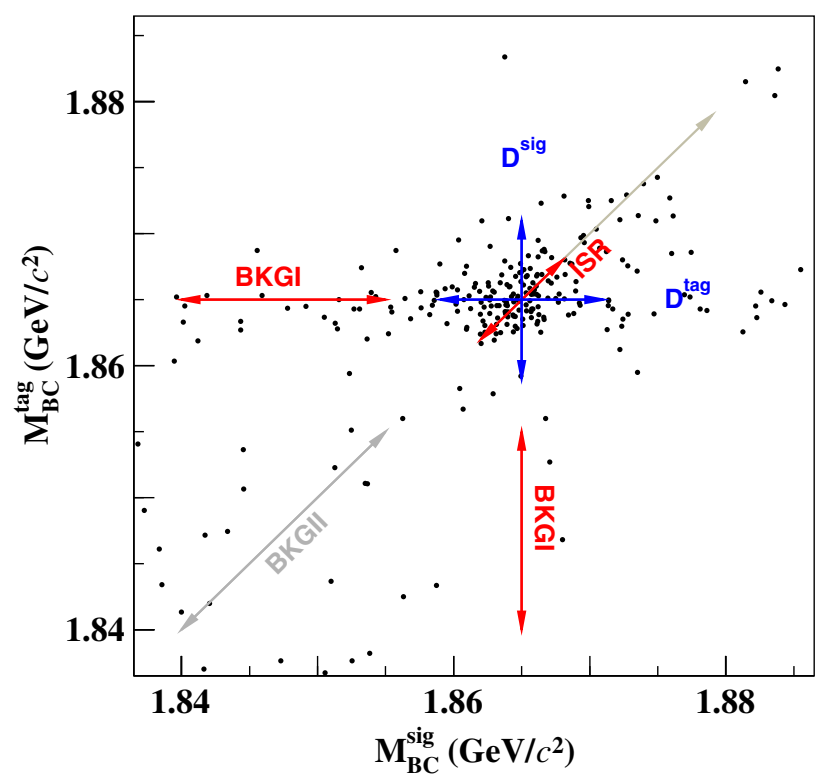

FIG. 2. The $M_{\mathrm{BC}}^{\mathrm{tag}}$ versus $M_{\mathrm{BC}}^{\mathrm{sig}}$ distribution of the accepted DT candidates of $D^{0} \rightarrow K^{+} K^{-} \pi^{0} \pi^{0}$ in data. Here, ISR denotes the signal spreading along the diagonal direction. 

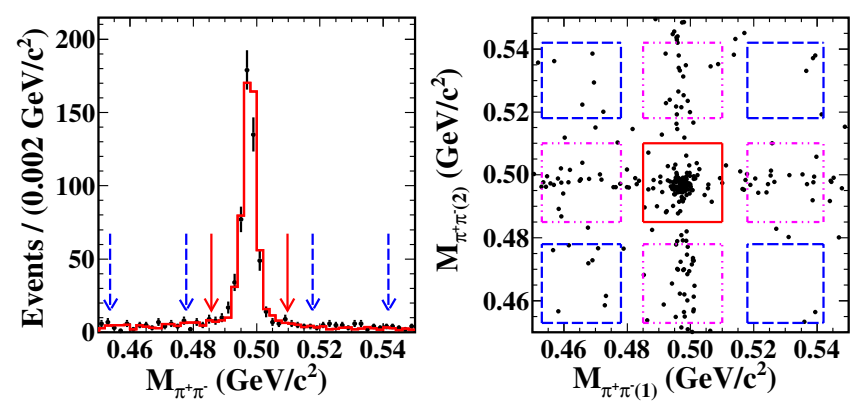

FIG. 3. (a) The $\pi^{+} \pi^{-}$invariant-mass distributions of the $D^{+} \rightarrow$ $K_{S}^{0} K^{-} \pi^{+} \pi^{+}$candidate events of data (points with error bars) and inclusive MC sample (histogram). Pairs of the red solid (blue dashed) arrows denote the $K_{S}^{0}$ signal (sideband) regions. (b) Distribution of $M_{\pi^{+} \pi^{-}(1)}$ versus $M_{\pi^{+} \pi^{-}(2)}$ for the $D^{0} \rightarrow K_{S}^{0} K_{S}^{0} \pi^{+} \pi^{-}$ candidate events in data. Red solid box denotes the 2D signal region. Pink dot-dashed (blue dashed) boxes indicate the $2 \mathrm{D}$ sideband 1 (2) regions.

BKGI for the $D^{0} \rightarrow K^{+} K^{-} \pi^{0} \pi^{0}$ signal decay is from $D^{0} \rightarrow \phi K_{S}^{0}\left(\rightarrow \pi^{0} \pi^{0}\right)$ with one fake $\pi^{0}$, which contribute $\sim 23 \%$ to the total horizonal BKGI.

For each signal $D$ decay mode, the yield of DT events $\left(N_{\mathrm{DT}}^{\mathrm{fit}}\right)$ is obtained from a two-dimensional (2D) unbinned maximum-likelihood fit [34] on the $M_{\mathrm{BC}}^{\mathrm{tag}}$ versus $M_{\mathrm{BC}}^{\mathrm{sig}}$ distribution of the accepted candidates. In the fit, the probability density functions (PDFs) of signal, BKGI, BKGII, and BKGIII are constructed as

(i) signal: $a(x, y)$,

(ii) BKGI: $b(x) \cdot c_{y}\left(y ; E_{\mathrm{b}}, \xi_{y}\right)+b(y) \cdot c_{x}\left(x ; E_{\mathrm{b}}, \xi_{x}\right)$,

(iii) BKGII: $c_{z}\left(z ; \sqrt{2} E_{\mathrm{b}}, \xi_{z}\right) \cdot g(k)$, and

(iv) BKGIII: $c_{x}\left(x ; E_{\mathrm{b}}, \boldsymbol{\xi}_{x}\right) \cdot c_{y}\left(y ; E_{\mathrm{b}}, \boldsymbol{\xi}_{y}\right)$,

respectively. Here, $x=M_{\mathrm{BC}}^{\mathrm{sig}}, y=M_{\mathrm{BC}}^{\mathrm{tag}}, z=(x+y) / \sqrt{2}$, and $k=(x-y) / \sqrt{2}$. The PDFs of signal $a(x, y), b(x)$, and $b(y)$ are described by the corresponding MC-simulated shapes. $c_{f}\left(f ; E_{\text {end }}, \xi_{f}\right)$ is an ARGUS function [33] defined above, where $f$ denotes $x, y$, or $z ; E_{\mathrm{b}}$ is fixed at $1.8865 \mathrm{GeV} . g(k)$ is a Gaussian function with mean of zero and standard deviation parametrized by $\sigma_{k}=$ $\sigma_{0} \cdot\left(\sqrt{2} E_{\mathrm{b}}-z\right)^{p}$, where $\sigma_{0}$ and $p$ are fit parameters.

Combinatorial $\pi^{+} \pi^{-}$pairs from the decays $D^{0} \rightarrow$ $K_{S}^{0} 2\left(\pi^{+} \pi^{-}\right)$[and $D^{0} \rightarrow 3\left(\pi^{+} \pi^{-}\right)$], $D^{0} \rightarrow K^{-} \pi^{+} \pi^{+} \pi^{-} \pi^{0}$, $D^{0} \rightarrow K^{+} \pi^{+} \pi^{-} \pi^{-} \pi^{0}, \quad D^{+} \rightarrow K^{-} \pi^{+} \pi^{+} \pi^{+} \pi^{-}, \quad D^{+} \rightarrow$ $K^{+} 2\left(\pi^{+} \pi^{-}\right), \quad D^{+} \rightarrow K^{+} \pi^{+} \pi^{-} \pi^{0} \pi^{0}, \quad D^{+} \rightarrow K_{S}^{0} \pi^{+} \pi^{+} \pi^{-} \pi^{0}$ [and $D^{+} \rightarrow 2\left(\pi^{+} \pi^{-}\right) \pi^{+} \pi^{0}$ ] may also satisfy the $K_{S}^{0}$ selection criteria and form peaking backgrounds around $M_{D}$ in the $M_{\mathrm{BC}}^{\mathrm{sig}}$ distributions for $D^{0} \rightarrow K_{S}^{0} K_{S}^{0} \pi^{+} \pi^{-}$, $D^{0} \rightarrow K_{S}^{0} K^{-} \pi^{+} \pi^{0}, D^{0} \rightarrow K_{S}^{0} K^{+} \pi^{-} \pi^{0}, D^{+} \rightarrow K_{S}^{0} K^{+} \pi^{0} \pi^{0}$ $D^{+} \rightarrow K_{S}^{0} K^{-} \pi^{+} \pi^{+}, \quad D^{+} \rightarrow K_{S}^{0} K^{+} \pi^{+} \pi^{-}, \quad$ and $D^{+} \rightarrow$ $K_{S}^{0} K_{S}^{0} \pi^{+} \pi^{0}$, respectively. This kind of peaking background is estimated by selecting events in the $K_{S}^{0}$ sideband region of $(0.454,0.478) \cup(0.518,0.542) \mathrm{GeV} / c^{2}$. For $D^{0} \rightarrow K_{S}^{0} K^{-} \pi^{+} \pi^{0}, D^{0} \rightarrow K_{S}^{0} K^{+} \pi^{-} \pi^{0}, D^{+} \rightarrow K_{S}^{0} K^{-} \pi^{+} \pi^{+}$, $D^{+} \rightarrow K_{S}^{0} K^{+} \pi^{+} \pi^{-}$, and $D^{+} \rightarrow K_{S}^{0} K^{+} \pi^{0} \pi^{0}$ decays, onedimensional (1D) signal and sideband regions are used. For $D^{0} \rightarrow K_{S}^{0} K_{S}^{0} \pi^{+} \pi^{-}$and $D^{+} \rightarrow K_{S}^{0} K_{S}^{0} \pi^{+} \pi^{0}$ decays, 2D signal and sideband regions are used. The $2 \mathrm{D} K_{S}^{0}$ signal region is defined as the square region with both $\pi^{+} \pi^{-}$ combinations lying in the $K_{S}^{0}$ signal regions. The $2 \mathrm{D} K_{S}^{0}$ sideband 1 (2) regions are defined as the square regions with 1 (2) $\pi^{+} \pi^{-}$combination(s) located in the $1 \mathrm{D} K_{S}^{0}$ sideband regions and the rest in the $1 \mathrm{D} K_{S}^{0}$ signal region. Figure 3 shows $1 \mathrm{D}$ and $2 \mathrm{D} \pi^{+} \pi^{-}$invariant-mass distributions as well as the $K_{S}^{0}$ signal and sideband regions.

For the signal decays involving $K_{S}^{0}$ meson(s) in the final states, the net yields of DT events are calculated by subtracting the sideband contribution from the DT fitted yield by
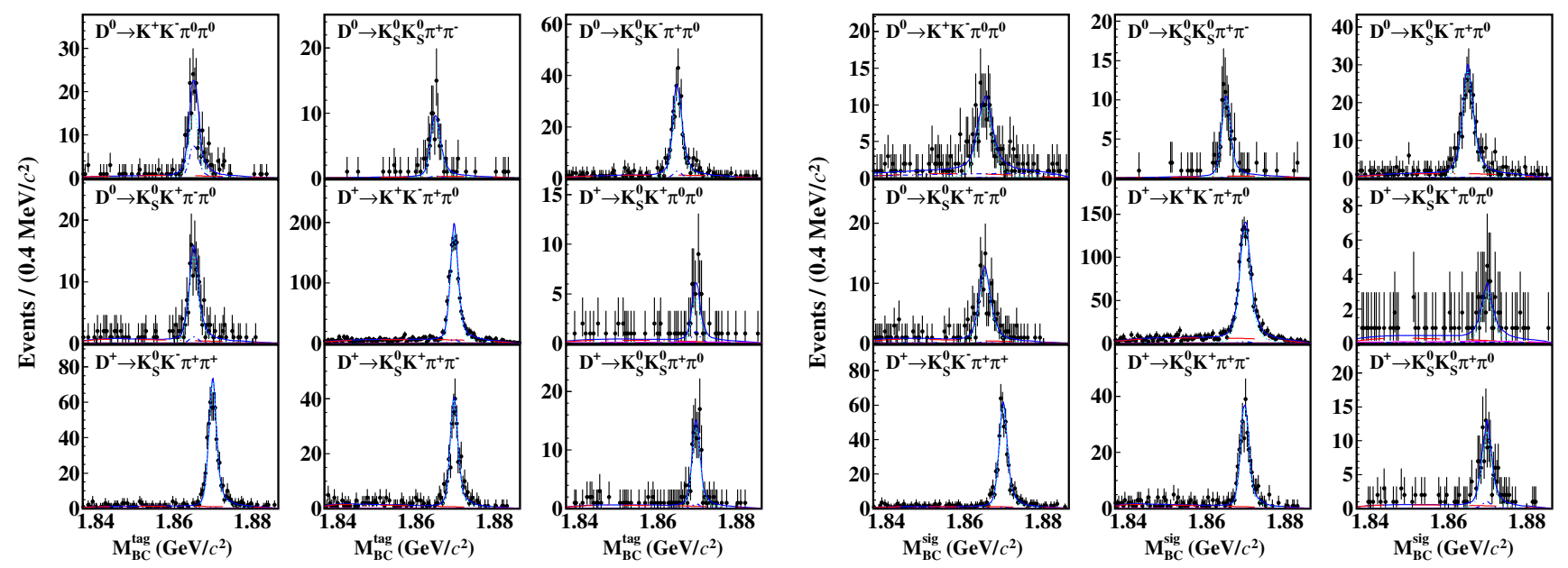

FIG. 4. Projections on the $M_{\mathrm{BC}}^{\mathrm{tag}}$ and $M_{\mathrm{BC}}^{\mathrm{sig}}$ distributions of the $2 \mathrm{D}$ fits to the DT candidate events with all $\bar{D}^{0}$ or $D^{-}$tags. Data are shown as points with error bars. Blue solid, light blue dotted, blue dot-dashed, red dot-long-dashed, and pink long-dashed curves denote the overall fit results, signal, BKGI, BKGII, and BKGIII components (see text), respectively. 

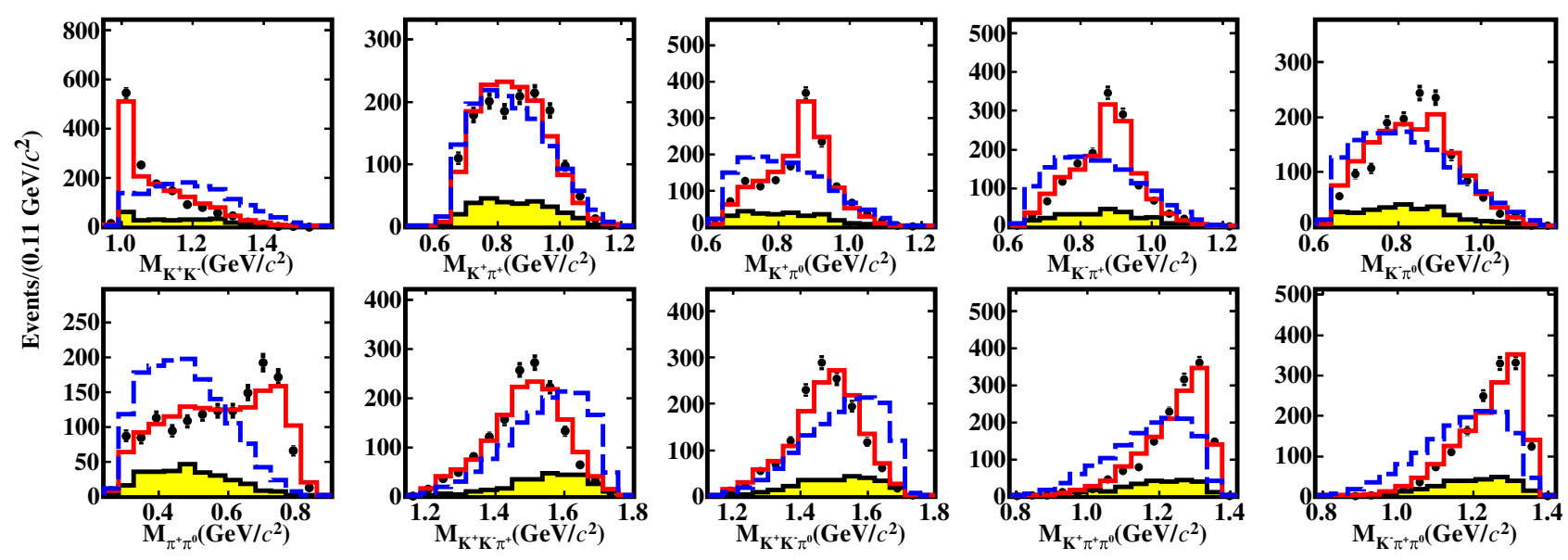

FIG. 5. The invariant mass distributions of two or three-body particle combinations of $D^{+} \rightarrow K^{+} K^{-} \pi^{+} \pi^{0}$ candidate events for data and MC simulations. Data are shown as points with error bars. Yellow hatched histograms are the backgrounds estimated from the inclusive MC sample. Red solid histograms are the mixed signal MC samples plus MC-simulated backgrounds. Blue dashed histograms are the PHSP signal MC samples plus MC-simulated backgrounds. Events have been required to be within $\left|M_{\mathrm{BC}}^{\mathrm{tag}(\operatorname{sig})}-M_{D}\right|<$ $0.006 \mathrm{GeV} / c^{2}$.

$N_{\mathrm{DT}}^{\mathrm{net}}=N_{\mathrm{DT}}^{\mathrm{fit}}-\Sigma_{i}^{N}\left[\left(-\frac{1}{2}\right)^{i+1} N_{\text {sid } i}^{\mathrm{fit}}\right]=N_{\mathrm{DT}}^{\mathrm{fit}}-\frac{1}{2} N_{K_{S}^{0}}^{\mathrm{sid}}$

Here, $N=1$ for the decays with one $K_{S}^{0}$ meson while $N=2$ for the decays with two $K_{S}^{0}$ mesons. The combinatorial $\pi^{+} \pi^{-}$ backgrounds are assumed to be uniformly distributed and double-counting is avoided by subtracting (2) yields from (1) yields appropriately. $N_{\mathrm{DT}}^{\mathrm{fit}}$ and $N_{\text {sid } i}^{\mathrm{fit}}$ are the fitted $D$ yields in the 1D or 2D signal region and sideband $i$ region, respectively. For the other signal decays, the net yields of DT events are $N_{\mathrm{DT}}^{\mathrm{fit}}$. Figure 4 shows the $M_{\mathrm{BC}}^{\mathrm{tag}}$ and $M_{\mathrm{BC}}^{\mathrm{sig}}$ projections of the 2D fits to data. From these 2D fits, we obtain the DTyields for individual signal decays as shown in Table I.

For each signal decay mode, the statistical significance is calculated according to $\sqrt{-2 \ln \left(\mathcal{L}_{0} / \mathcal{L}_{\max }\right)}$, where $\mathcal{L}_{\max }$ and $\mathcal{L}_{0}$ are the maximum likelihoods of the fits with and without involving the signal component, respectively. The effect of combinatorial $\pi^{+} \pi^{-}$backgrounds in the $K_{S}^{0}$-signal regions has been considered for the decays involving a $K_{S}^{0}$. The statistical significance for each signal decay is found to be greater than $8 \sigma$.

\section{RESULTS}

Each of the $D^{0} \rightarrow K_{S}^{0} K^{-} \pi^{+} \pi^{0}, D^{+} \rightarrow K^{+} K^{-} \pi^{+} \pi^{0}$, $D^{+} \rightarrow K_{S}^{0} K^{-} \pi^{+} \pi^{+}$, and $D^{+} \rightarrow K_{S}^{0} K^{+} \pi^{+} \pi^{-}$decays is modeled by the corresponding mixed signal MC samples, in which the dominant decay modes containing resonances of $K^{*}(892), \rho(770)$, and $\phi$ are mixed with the phase space (PHSP) signal MC samples. The mixing ratios are determined by examining the corresponding invariant mass and momentum spectra. The other decays, which are limited in statistics, are generated with the PHSP generator. The momentum and the polar angle distributions of the daughter particles and the invariant masses of each two- and three-body particle combinations of the data agree with those of the MC simulations. As an example, Fig. 5 shows the invariant mass distributions of two- or three-body particle combinations of $D^{+} \rightarrow K^{+} K^{-} \pi^{+} \pi^{0}$ candidate events for data and MC simulations.

The measured values of $N_{\mathrm{DT}}^{\text {net }}, \epsilon_{\text {sig }}$, and the obtained BFs are summarized in Table I. The current world-average values are also given for comparison. The signal efficiencies have been corrected by the necessary data-MC differences in the selection efficiencies of $K^{ \pm}$and $\pi^{ \pm}$ tracking and PID procedures and the $\pi^{0}$ reconstruction. These efficiencies include the BFs of the $K_{S}^{0}$ and $\pi^{0}$ decays, obtained by removing $K_{S}^{0}$ sideband contribution. The difference of efficiencies with and without removing $K_{S}^{0}$ sideband contribution is $(2-3) \%$.

The efficiencies for $D^{0} \rightarrow K_{S}^{0} K^{-} \pi^{+} \pi^{0}$ and $D^{+} \rightarrow$ $K_{S}^{0} K^{+} \pi^{+} \pi^{-}$before $K_{S}^{0}(\omega)$ rejection are $(8.23 \pm 0.07) \%$ and $(10.89 \pm 0.07) \%$, respectively. Our nominal efficiency for $D^{+} \rightarrow K_{S}^{0} K^{+} \pi^{+} \pi^{-}\left(D^{0} \rightarrow K_{S}^{0} K^{-} \pi^{+} \pi^{0}\right)$ is lower than that of $D^{+} \rightarrow K_{S}^{0} K^{-} \pi^{+} \pi^{+} \quad\left(D^{0} \rightarrow K_{S}^{0} K^{+} \pi^{-} \pi^{0}\right)$ due to different intermediate resonant states and further $K_{S}^{0}(\omega)$ rejection in the $\pi^{+} \pi^{-}\left(K_{S}^{0} \pi^{0}\right)$ mass spectrum.

\section{SYSTEMATIC UNCERTAINTIES}

The systematic uncertainties are estimated relative to the measured BFs and are discussed below. In BF determinations using Eq. (1), all uncertainties associated with the selection of tagged $\bar{D}$ canceled in the ratio. The systematic uncertainties in the total yields of ST $\bar{D}$ mesons related to the $M_{\mathrm{BC}}$ fits to the ST $\bar{D}$ candidates, were previously 
estimated to be $0.5 \%$ for both neutral and charged $\bar{D}$ [22-24].

The tracking and PID efficiencies for $K^{ \pm}$or $\pi^{ \pm}$, $\epsilon_{K \text { or } \pi}^{\text {trang(PID) }}\left[\right.$ data] and $\epsilon_{K \text { or } \pi}^{\text {tracing(PID) }}[\mathrm{MC}]$, are investigated using DT $D \bar{D}$ hadronic events. The averaged ratios between data and MC efficiencies $\left(f_{K \text { or } \pi}^{\text {tracking(PID) }}=\epsilon_{K \text { or } \pi}^{\text {trang(PID) }}[\right.$ data $] /$ $\epsilon_{K \text { or } \pi}^{\text {trang(PID) }}[\mathrm{MC}]$ ) of tracking (PID) for $K^{ \pm}$or $\pi^{ \pm}$are weighted by the corresponding momentum spectra of signal MC events, giving $f_{K}^{\text {tracking }}$ to be $1.022-1.031$ and $f_{\pi}^{\text {tracking }}$ to be close to unity. After correcting the MC efficiencies by $f_{K}^{\text {tracking }}$, the residual uncertainties of $f_{K \text { or } \pi}^{\text {trang }}$ are assigned as the systematic uncertainties of tracking efficiencies, which are (0.4-0.7)\% per $K^{ \pm}$and $(0.2-0.3) \%$ per $\pi^{ \pm} . f_{K}^{\mathrm{PID}}$ and $f_{\pi}^{\mathrm{PID}}$ are all close to unity and their individual uncertainties, $(0.2-0.3) \%$, are taken as the associated systematic uncertainties per $K^{ \pm}$or $\pi^{ \pm}$.

The systematic error related to the uncertainty in the $K_{S}^{0}$ reconstruction efficiency is estimated from measurements of $J / \psi \rightarrow K^{*}(892)^{\mp} K^{ \pm}$and $J / \psi \rightarrow \phi K_{S}^{0} K^{ \pm} \pi^{\mp}$ control samples [35] and found to be $1.6 \%$ per $K_{S}^{0}$. The systematic uncertainty of $\pi^{0}$ reconstruction efficiency is assigned as $(0.7-0.8) \%$ per $\pi^{0}$ from a study of DT $D \bar{D}$ hadronic decays of $\bar{D}^{0} \rightarrow K^{+} \pi^{-} \pi^{0}$ and $\bar{D}^{0} \rightarrow K_{S}^{0} \pi^{0}$ decays tagged by either $D^{0} \rightarrow K^{-} \pi^{+}$or $D^{0} \rightarrow K^{-} \pi^{+} \pi^{+} \pi^{-}[22,23]$.

The systematic uncertainty in the $2 \mathrm{D}$ fit to the $M_{\mathrm{BC}}^{\mathrm{tag}}$ versus $M_{\mathrm{BC}}^{\mathrm{sig}}$ distribution is examined via the repeated measurements in which the signal shape and the endpoint of the ARGUS function $\left( \pm 0.2 \mathrm{MeV} / c^{2}\right)$ are varied. Quadratically summing the changes of the BFs for these two sources yields the corresponding systematic uncertainties.
The systematic uncertainty due to the $\Delta E_{\text {sig }}$ requirement is assigned to be $0.3 \%$, which corresponds to the largest efficiency difference with and without smearing the dataMC Gaussian resolution of $\Delta E_{\text {sig }}$ for signal MC events. Here, the smeared Gaussian parameters are obtained by using the samples of DT events $D^{0} \rightarrow K_{S}^{0} \pi^{0}, D^{0} \rightarrow$ $K^{-} \pi^{+} \pi^{0}, D^{0} \rightarrow K^{-} \pi^{+} \pi^{0} \pi^{0}$, and $D^{+} \rightarrow K^{-} \pi^{+} \pi^{+} \pi^{0}$ versus the same $\bar{D}$ tags in our nominal analysis. The systematic uncertainties due to $K_{S}^{0}$ sideband choice and $K_{S}^{0}$ rejection mass window are assigned by examining the changes of the BFs via varying nominal $K_{S}^{0}$ sideband and corresponding rejection window by $\pm 5 \mathrm{MeV} / c^{2}$.

For the decays whose efficiencies are estimated with mixed signal MC events, the systematic uncertainty in the MC modeling is determined by comparing the signal efficiency when changing the percentage of MC sample components. For the decays whose efficiencies are estimated with PHSP-distributed signal MC events, the uncertainties are assigned as the change of the signal efficiency after adding the possible decays containing $K^{*}(892)$ or $\rho(770)$. The imperfect simulations of the momentum and $\cos \theta$ distributions of charged particles are considered as a source of systematic uncertainty. The signal efficiencies are reweighted by those distributions in data with background subtracted. The largest change of the reweighted to nominal efficiencies, $0.9 \%$, is assigned as the corresponding systematic uncertainty.

The measurements of the BFs of the neutral $D$ decays are affected by quantum correlation effect. For each neutral $D$ decay, the $C P$-even component is estimated by the $C P$ even tag $D^{0} \rightarrow K^{+} K^{-}$and the $C P$-odd tag $D^{0} \rightarrow K_{S}^{0} \pi^{0}$. Using the same method as described in Ref. [36] and the

TABLE II. Systematic uncertainties (\%) in the measurements of the BFs of the signal decays (1) $D^{0} \rightarrow K^{+} K^{-} \pi^{0} \pi^{0}$, (2) $D^{0} \rightarrow K_{S}^{0} K_{S}^{0} \pi^{+} \pi^{-}$, (3) $D^{0} \rightarrow K_{S}^{0} K^{-} \pi^{+} \pi^{0}$, (4) $D^{0} \rightarrow K_{S}^{0} K^{+} \pi^{-} \pi^{0}$, (5) $D^{+} \rightarrow K^{+} K^{-} \pi^{+} \pi^{0}$, (6) $D^{+} \rightarrow K_{S}^{0} K^{+} \pi^{0} \pi^{0}$, (7) $D^{+} \rightarrow K_{S}^{0} K^{-} \pi^{+} \pi^{+}$, (8) $D^{+} \rightarrow K_{S}^{0} K^{+} \pi^{+} \pi^{-}$, and (9) $D^{+} \rightarrow K_{S}^{0} K_{S}^{0} \pi^{+} \pi^{0}$.

\begin{tabular}{|c|c|c|c|c|c|c|c|c|c|}
\hline Source/Signal decay & 1 & 2 & 3 & 4 & 5 & 6 & 7 & 8 & 9 \\
\hline$N_{\mathrm{ST}}^{\text {tot }}$ & 0.5 & 0.5 & 0.5 & 0.5 & 0.5 & 0.5 & 0.5 & 0.5 & 0.5 \\
\hline$(K / \pi)^{ \pm}$tracking & 1.0 & 0.6 & 0.9 & 0.9 & 1.6 & 0.4 & 1.1 & 1.2 & 0.3 \\
\hline$(K / \pi)^{ \pm} \mathrm{PID}$ & 0.4 & 0.4 & 0.6 & 0.6 & 1.0 & 0.2 & 0.6 & 0.7 & 0.2 \\
\hline$K_{S}^{0}$ reconstruction & $\ldots$ & 3.2 & 1.6 & 1.6 & $\ldots$ & 1.6 & 1.6 & 1.6 & 3.2 \\
\hline$\pi^{0}$ reconstruction & 1.6 & $\ldots$ & 0.7 & 0.7 & 0.8 & 1.6 & $\ldots$ & $\ldots$ & 0.7 \\
\hline$\Delta E_{\text {sig }}$ requirement & 0.7 & 0.7 & 0.7 & 0.7 & 0.7 & 0.7 & 0.7 & 0.7 & 0.7 \\
\hline$K_{S}^{0}$ rejection & 4.2 & 2.4 & $\ldots$ & $\ldots$ & $\ldots$ & 4.2 & $\ldots$ & 0.8 & $\ldots$ \\
\hline$K_{S}^{0}$ sideband & $\ldots$ & 0.2 & 1.1 & 0.2 & $\ldots$ & 1.3 & 0.1 & 0.1 & 0.2 \\
\hline Quoted BFs & 0.0 & 0.1 & 0.1 & 0.1 & 0.0 & 0.1 & 0.1 & 0.1 & 0.1 \\
\hline MC statistics & 0.8 & 0.6 & 0.7 & 0.6 & 0.5 & 0.4 & 0.4 & 0.5 & 0.6 \\
\hline MC modeling & 1.3 & 1.0 & 0.5 & 0.7 & 2.1 & 1.4 & 0.5 & 0.7 & 0.5 \\
\hline Imperfect simulation & 0.9 & 0.9 & 0.9 & 0.9 & 0.9 & 0.9 & 0.9 & 0.9 & 0.9 \\
\hline$D \bar{D}$ opening angle & 0.4 & 0.4 & 0.4 & 0.4 & 0.4 & 0.4 & 0.4 & 0.4 & 0.4 \\
\hline $2 \mathrm{D}$ fit & 1.3 & 2.8 & 3.1 & 1.5 & 1.9 & 2.7 & 0.5 & 0.6 & 3.0 \\
\hline Quantum correlation effect & 1.6 & 2.8 & 3.4 & 1.1 & $\ldots$ & $\ldots$ & $\ldots$ & $\ldots$ & $\ldots$ \\
\hline Total & 5.4 & 5.9 & 5.4 & 3.2 & 3.8 & 6.0 & 2.5 & 2.8 & 4.7 \\
\hline
\end{tabular}


necessary parameters quoted from Refs. [37-39], we find the correction factors to account for the quantum correlation effect on the measured BFs are $\left(98.3_{-1.1 \text { stat }}^{+1.6}\right) \%$, $\left(98.1_{-1.7 \text { stat }}^{+2.8}\right) \%, \quad\left(95.9_{-2.7 \text { stat }}^{+3.4}\right) \%$, and $\left(98.4_{-1.0 \text { stat }}^{+1.1}\right) \%$ for $D^{0} \rightarrow K^{+} K^{-} \pi^{0} \pi^{0}, D^{0} \rightarrow K_{S}^{0} K_{S}^{0} \pi^{+} \pi^{-}, D^{0} \rightarrow K_{S}^{0} K^{-} \pi^{+} \pi^{0}$, and $D^{0} \rightarrow K_{S}^{0} K^{+} \pi^{-} \pi^{0}$, respectively. After correcting the signal efficiencies by the individual factors, the residual uncertainties are assigned as systematic uncertainties.

The uncertainties due to the limited MC statistics for various signal decays, (0.4-0.8)\%, are taken into account as a systematic uncertainty. The uncertainties of the quoted BFs of the $K_{S}^{0} \rightarrow \pi^{+} \pi^{-}$and $\pi^{0} \rightarrow \gamma \gamma$ decays are $0.07 \%$ and $0.03 \%$, respectively [9].

The efficiencies of $D \bar{D}$ opening angle requirement is studied by using the DT events of $D^{0} \rightarrow K^{-} \pi^{+} \pi^{+} \pi^{-}$, $D^{0} \rightarrow K^{-} \pi^{+} \pi^{0} \pi^{0}$, and $D^{0} \rightarrow K^{-} \pi^{+} \pi^{0}$ tagged by the same tag modes in our nominal analysis. The largest difference of the accepted efficiencies between data and MC simulations, $0.4 \%$, is assigned as the associated systematic uncertainty.

Table II summarizes the systematic uncertainties in the BF measurements. For each signal decay, the total systematic uncertainty is obtained by adding the above effects in quadrature to be $(2.5-6.0) \%$ for various signal decay modes.

\section{SUMMARY}

In summary, by analyzing a data sample obtained in $e^{+} e^{-}$collisions at $\sqrt{s}=3.773 \mathrm{GeV}$ with the BESIII detector and corresponding to an integrated luminosity of $2.93 \mathrm{fb}^{-1}$, we obtained the first direct measurements of the absolute BFs of nine $D^{0(+)} \rightarrow K \bar{K} \pi \pi$ decays containing $K_{S}^{0} \quad$ or $\pi^{0}$ mesons. The $D^{0} \rightarrow K^{+} K^{-} \pi^{0} \pi^{0}, \quad D^{0} \rightarrow$ $K_{S}^{0} K^{-} \pi^{+} \pi^{0}, D^{0} \rightarrow K_{S}^{0} K^{+} \pi^{-} \pi^{0}, D^{+} \rightarrow K_{S}^{0} K^{+} \pi^{0} \pi^{0}$, and $D^{+} \rightarrow K_{S}^{0} K_{S}^{0} \pi^{+} \pi^{0}$ decays are observed for the first time. Compared to the world-average values, the BFs of the $D^{0} \rightarrow K_{S}^{0} K_{S}^{0} \pi^{+} \pi^{-}, D^{+} \rightarrow K^{+} K^{-} \pi^{+} \pi^{0}, D^{+} \rightarrow K_{S}^{0} K^{-} \pi^{+} \pi^{+}$, and $D^{+} \rightarrow K_{S}^{0} K^{+} \pi^{+} \pi^{-}$decays are measured with improved precision. Our BFs of $D^{+} \rightarrow K_{S}^{0} K^{-} \pi^{+} \pi^{+}$and $D^{+} \rightarrow K_{S}^{0} K^{+} \pi^{+} \pi^{-}$are in agreement with individual world averages within $1 \sigma$ while our BFs of $D^{0} \rightarrow K_{S}^{0} K_{S}^{0} \pi^{+} \pi^{-}$and $D^{+} \rightarrow K^{+} K^{-} \pi^{+} \pi^{0}$ deviate with individual world averages by $2.3 \sigma$ and $2.8 \sigma$, respectively. The precision of the $\mathrm{BF}$ of $D^{+} \rightarrow K^{+} K^{-} \pi^{+} \pi^{0}$ is improved by a factor of about seven. Future amplitude analyses of all these $D^{0(+)} \rightarrow K \bar{K} \pi \pi$ decays with larger data samples foreseen at BESIII [40], Belle II [41], and LHCb [42] will supply rich information of the two-body decay modes containing scalar, vector, axial and tensor mesons, thereby benefiting the understanding of quark SU(3)-flavor symmetry.

\section{ACKNOWLEDGMENTS}

The authors are thankful for valuable discussions with Prof. Fu-sheng Yu. The BESIII collaboration thanks the staff of BEPCII and the IHEP computing center for their strong support. This work is supported in part by National Key Basic Research Program of China under Contract No. 2015CB856700; National Natural Science Foundation of China (NSFC) under Contracts No. 11775230, No. 11475123, No. 11625523, No. 11635010, No. 11735014, No. 11822506, No. 11835012, No. 11935015, No. 11935016, No. 11935018, No. 11961141012; the Chinese Academy of Sciences (CAS) Large-Scale Scientific Facility Program; Joint Large-Scale Scientific Facility Funds of the NSFC and CAS under Contracts No. U1532101, No. U1932102, No. U1732263, No. U1832207; CAS Key Research Program of Frontier Sciences under Contracts No. QYZDJ-SSW-SLH003, No. QYZDJ-SSW-SLH040; 100 Talents Program of CAS; INPAC and Shanghai Key Laboratory for Particle Physics and Cosmology; ERC under Contract No. 758462; German Research Foundation DFG under Contracts Nos. Collaborative Research Center CRC 1044, FOR 2359; Istituto Nazionale di Fisica Nucleare, Italy; Ministry of Development of Turkey under Contract No. DPT2006K120470; National Science and Technology fund; STFC (United Kingdom); The Knut and Alice Wallenberg Foundation (Sweden) under Contract No. 2016.0157; The Royal Society, UK under Contracts No. DH140054, No. DH160214; The Swedish Research Council; U.S. Department of Energy under Contracts No. DE-FG0205ER41374, No. DE-SC-0012069.
[1] H. N. Li, C. D. Lü, and F. S. Yu, Phys. Rev. D 86, 036012 (2012).

[2] H. Y. Cheng and C. W. Chiang, Phys. Rev. D 81, 074021 (2010).

[3] Q. Qin, H. N. Li, C. D. Lü, and F. S. Yu, Phys. Rev. D 89, 054006 (2014).
[4] H. Y. Cheng, Phys. Rev. D 67, 094007 (2003); 68, 014015 (2003).

[5] P. F. Guo, D. Wang, and F. S. Yu, Nucl. Phys. Rev. 36, 125 (2019).

[6] X. W. Kang and H. B. Li, Phys. Lett. B 684, 137 (2010). 
[7] J. Charles, S. Descotes-Genon, X. W. Kang, H. B. Li, and G. R. Lu, Phys. Rev. D 81, 054032 (2010).

[8] M. Saur and F. S. Yu, Sci. Bull. 65, 1428 (2020).

[9] P. A. Zyla et al. (Particle Data Group), Prog. Theor. Exp. Phys. 2020, 083C01 (2020).

[10] H. Albrecht et al. (ARGUS Collaboration), Z. Phys. C 64, 375 (1994).

[11] J. M. Link et al. (FOCUS Collaboration), Phys. Lett. B 607, 59 (2005).

[12] J. M. Link et al. (FOCUS Collaboration), Phys. Rev. Lett. 87, 162001 (2001).

[13] S. Barlag et al. (ACCMOR Collaboration), Z. Phys. C 55, 383 (1992).

[14] M. Ablikim et al. (BESIII Collaboration), Chin. Phys. C 37, 123001 (2013); Phys. Lett. B 753, 629 (2016).

[15] M. Ablikim et al. (BESIII Collaboration), Nucl. Instrum. Methods Phys. Res., Sect. A 614, 345 (2010).

[16] C. H. Yu et al., in Proc. IPAC2016 (Busan, Korea, 2016).

[17] S. Agostinelli et al. (GEANT4 Collaboration), Nucl. Instrum. Methods Phys. Res., Sect. A 506, 250 (2003).

[18] S. Jadach, B. F. L. Ward, and Z. Was, Comput. Phys. Commun. 130, 260 (2000); Phys. Rev. D 63, 113009 (2001).

[19] D. J. Lange, Nucl. Instrum. Methods Phys. Res., Sect. A 462, 152 (2001); R. G. Ping, Chin. Phys. C 32, 599 (2008).

[20] J. C. Chen, G. S. Huang, X. R. Qi, D. H. Zhang, and Y. S. Zhu, Phys. Rev. D 62, 034003 (2000).

[21] E. Richter-Was, Phys. Lett. B 303, 163 (1993).

[22] M. Ablikim et al. (BESIII Collaboration), Eur. Phys. J. C 76, 369 (2016).

[23] M. Ablikim et al. (BESIII Collaboration), Chin. Phys. C 40, 113001 (2016).

[24] M. Ablikim et al. (BESIII Collaboration), Phys. Rev. Lett. 121, 171803 (2018).

[25] M. Ablikim et al. (BESIII Collaboration), Phys. Rev. Lett. 123, 231801 (2019).
[26] M. Ablikim et al. (BESIII Collaboration), Phys. Rev. D 101, 052009 (2020).

[27] M. Ablikim et al. (BESIII Collaboration), Phys. Rev. D 101, 072005 (2020).

[28] M. Ablikim et al. (BESIII Collaboration), Phys. Rev. Lett. 124, 231801 (2020).

[29] M. Ablikim et al. (BESIII Collaboration), Phys. Rev. Lett. 124, 241803 (2020).

[30] M. Ablikim et al. (BESIII Collaboration), arXiv: 2007.07674 [Phys. Rev. Lett. (to be published)].

[31] M. Ablikim et al. (BESIII Collaboration), arXiv: 2008.05754 [Phys. Rev. D (to be published)].

[32] M. Ablikim et al. (BESIII Collaboration), Phys. Lett. B 734, 227 (2014).

[33] H. Albrecht et al. (ARGUS Collaboration), Phys. Lett. B 241, 278 (1990).

[34] S. Dobbs et al. (CLEO Collaboration), Phys. Rev. D 76, 112001 (2007).

[35] M. Ablikim et al. (BESIII Collaboration), Phys. Rev. D 92, 112008 (2015).

[36] M. Ablikim et al. (BESIII Collaboration), Phys. Rev. D 100, 072006 (2019).

[37] T.Gershon, J. Libby, and G. Wilkinson, Phys. Lett. B 750, 338 (2015).

[38] T. Evans, S. T. Harnew, J. Libby, S. Malde, J. Rademacker, and G. Wilkinson, Phys. Lett. B 757, 520 (2016); 765, 402(E) (2017).

[39] Heavy Flavor Averaging Group (HFLAV), http://www.slac .stanford.edu/xorg/hflav/charm/.

[40] M. Ablikim et al. (BESIII Collaboration), Chin. Phys. C 44, 040001 (2020).

[41] E. Kou et al. (Belle II Collaboration), Prog. Theor. Exp. Phys. 2019, 123C01 (2019).

[42] R. Aaij et al. (LHCb Collaboration), arXiv:1808.08865. 